\title{
Pronounced Effects of Interannular Trimethylene Bridges on the Intramolecular Electron-Transfer Rate in Mixed-Valence Biferrocenium Salts: Valence-Trapped Electronic Structure with Unusual Structural and Mössbauer Characteristics
}

\author{
Teng-Yuan Dong, ,'1 Ting-Yu Lee, ${ }^{1 \mathrm{a}}$ Shu-Hwei Lee, ${ }^{1 \mathrm{~b}}$ Gene-Hsiang Lee, ${ }^{2}$ and \\ Shie-Ming Peng ${ }^{2}$ \\ Department of Chemistry, National Sun Yat-sen University, Kaohsuing, Taiwan, ROC, \\ the Institute of Chemistry, Academia Sinica, Nankang, Taipei, Taiwan, ROC, and \\ Department of Chemistry, National Taiwan University, Taipei, Taiwan, ROC
}

Received February 22, $1994^{\circledR}$

\begin{abstract}
The X-ray structure determinations of two new constitutional isomers of mixed-valence biferrocenium salts and the effects of interannular trimethylene bridges on intramolecular electron-transfer rates in both solid and solution states are reported. The X-ray structure of $1^{\prime}, 2: 3^{\prime}, 4: 1^{\prime \prime \prime}, 2^{\prime \prime}: 3^{\prime \prime \prime}, 4^{\prime \prime}$-tetrakis(propane-1,3-diyl)-1,1"1-biferrocene (17a) has been determined at $298 \mathrm{~K}: P \overline{1}, a=9.144(1) \AA, b=15.051(2) \AA, c=17.844(4) \AA, \alpha=106.39(3)^{\circ}, \beta=91.73(2)^{\circ}, \gamma$ $=90.76(2)^{\circ}, Z=4, D_{\mathrm{c}}=1.496 \mathrm{~g} \mathrm{~cm}^{-3}, R_{F}=0.040$, and $R_{\mathrm{wF}}=0.046$. The isomeric compound $17 \mathrm{~b}$ crystallizes in the triclinic space group $P \overline{1}$ with eight molecules in a unit cell with dimensions $a=9.209(1) \AA, b=24.132(2) \AA, c=24.501(4) \AA, \alpha=116.808(9)^{\circ}, \beta=87.98(1)^{\circ}, \gamma=91.58(1)^{\circ}$, $D_{\mathrm{c}}=1.451 \mathrm{~g} \mathrm{~cm}^{-3}, R_{F}=0.038$, and $R_{\mathrm{w} F}=0.041$. The mixed-valence compound $1^{\prime}, 2: 3^{\prime}, 4: 1^{\prime \prime \prime}, 2^{\prime \prime}$ : $3^{\prime \prime \prime}, 4^{\prime \prime}$-tetrakis (propane-1,3-diyl)-1,1"'-biferrocenium triiodide (18a) crystallizes in the monoclinic space group $P 2_{1} / c$ with $a=11.363(2) \AA, b=13.666(2) \AA, c=19.753(3) \AA, \beta=104.793(12)^{\circ}, Z$ $=4, D_{\mathrm{c}}=2.04 \mathrm{~g} \mathrm{~cm}^{-3}, R_{F}=0.039$, and $R_{\mathrm{w} F}=0.043$. The isomeric mixed-valence compound $18 \mathrm{~b}$ crystallizes in the monoclinic space group $P 2_{1} / n$ with $a=11.578(1) \AA, b=12.108$ (2) $\AA, c=$ $21.162(3) \AA, \beta=90.379^{\circ}, Z=4, D_{\mathrm{c}}=2.04 \mathrm{~g} \mathrm{~cm}^{-3}, R_{F}=0.030$, and $R_{\mathrm{wF}}=0.032$. In common with most mixed-valence compounds, compounds $18 \mathrm{a}$ and $18 \mathrm{~b}$ in $\mathrm{CH}_{2} \mathrm{Cl}_{2}$ have intervalence transition (IT) bands at 5050 and $4808 \mathrm{~cm}^{-1}$, respectively. The corresponding rate constants of intramolecular electron-transfer rates for $18 \mathrm{a}, \mathrm{b}$ are $2.31 \times 10^{12}$ and $3.26 \times 10^{12} \mathrm{~s}^{-1}$, respectively. The Mössbauer results indicate that compounds $18 \mathrm{a}, \mathrm{b}$ have valence-trapped electronic structures (electron-transfer rate less than $\sim 10^{7} \mathrm{~s}^{-1}$ in solid state). We believe that the most important factor controlling the difference of intramolecular electron-transfer rates between the solution state and the solid state is the degree of coplanarity between the two $\mathrm{Cp}$ rings in the fulvalenide bridge. We also demonstrate that the perturbations caused by the interannular trimethylene bridge have dramatic influences on the electronic structure in a series of model ferrocenium salts.
\end{abstract}

\section{Introduction}

There has been considerable progress made in understanding the factors which control the rate of intramolecular electron transfer in the solid state for mixed-valence compounds. ${ }^{3}$ The rates of electron transfer in mixedvalence cations 1-13 (Chart 1) can be sensitively controlled by environmental factors. ${ }^{4-24}$ Compounds 2-5 give unusual temperature-dependent Mössbauer spectra. ${ }^{5-8}$ At tem-

\footnotetext{
- Abstract published in Advance ACS Abstracts, April 15, 1994.
}

(1) (a) Academia Sinica. (b) National Sun Yat-sen University.

(2) National Taiwan University.

(3) For reviews, see: (a) Day, P. Int. Rev. Phys. Chem. 1981, 1, 149. (b) Brown, D. B., Ed. Mixed-Valence Compounds, Theory and Applications in Chemistry, Physics, Geology, and Biology; Reidel: Boston, MA, 1980. (c) Creutz, C. Prog. Inorg. Chem. 1983, 30, 1-73. (d) Richardson, D. E.; Taube, H. Coord. Chem. Rev. 1984, 60, 107.

(4) Hendrickson, D. N.;Oh, S. M.; Dong, T.-Y.; Moore, M. F. Comments Inorg. Chem. 1985, 4, 329 .

(5) Dong, T.-Y.; Cohn, M. J.; Hendrickson, D. N.; Pierpont, C. G. J. Am. Chem. Soc. 1985, 107, 4777.

(6) Cohn, M.J.; Dong, T.-Y.; Hendrickson, D. N.; Geib, S. J.; Rheingold, A. L. J. Chem. Soc., Chem. Commun. 1985, 1095.

(7) Dong, T.-Y.; Hendrickson, D. N.; Iwai, K.; Cohn, M. J.; Rheingold, A. L.; Sano, H.; Motoyama, S. J. Am. Chem. Soc. 1985, $107,7996$.

(8) lijima, S.; Saida, R.; Motoyama, I.; Sano, H. Bull. Chem. Soc. Jpn. $1981,54,1375$. peratures below $200 \mathrm{~K}$ they show two doublets, one for the $\mathrm{Fe}^{\mathrm{II}}$ and the other for the $\mathrm{Fe}^{\mathrm{III}}$ sites. Increasing the sample temperature in each case causes the two doublets to move

(9) Nakashima, S.; Masuda, Y.; Motoyama, I.; Sano, H. Bull. Chem. Soc. Jpn. 1987, 60, 1673 .

(10) Nakashima, S.; Katada, M.; Motoyama, I.; Sano, H. Bull. Chem. Soc. Jpn. 1987, 60, 2253.

(11) Dong, T.-Y.; Kambara, T.; Hendrickson, D. N. J. Am. Chem. Soc. $1986,108,4423$.

(12) Dong, T.-Y.; Kambara, T.; Hendrickson, D. N. J. Am. Chem. Soc. $1986,108,5857$.

(13) Sorai, M.; Nishimori, A.; Hendrickson, D. N.; Dong, T.-Y.; Cohn, M. J. J. Am. Chem. Soc. 1987, $109,4266$.

(14) Kambara, T.; Hendrickson, D. N.; Dong, T.-Y.; Cohn, M. J. J. Chem. Phys. 1987, 86, 2326.

(15) Konno, M.; Hyodo, S.; Iijima, S. Bull. Chem. Soc. Jpn. 1982, 55, 2327.

(16) Wong, K. Y.; Schatz, P. N. Prog. Inorg. Chem. 1981, 28, 369.

(17) Dong, T.-Y.; Hwang, M. Y.; Schei, C. C.; Peng, S. M.; Yeh, S. K. J. Organomet. Chem. 1989, 369, C33.

(18) Dong, T.-Y.; Schei, C. C.; Hwang, M. Y.; Lee, T. Y.; Yeh, S. K.; Wen, Y. S. Organometallics 1992, 11, 573.

(19) Kai, M.; Katada, M.; Sano, H. Chem. Lett. 1988, 1523.

(20) Dong, T.-Y.; Schei, C. C.; Hsu, T. L.; Lee, S. L.; Li, S. J. Inorg. Chem. 1991, 30, 2457.

(21) Dong, T.-Y.; Chou, C. Y. J. Chem. Soc., Chem. Commun. 1990 , 1332.

(22) Webb, R. J.; Geib, S. J.; Staley, D. L.; Rheingold, A. L.; Hendrickson, D. N. J. Am. Chem. Soc. 1990, 112, 5031. 
Chart 1
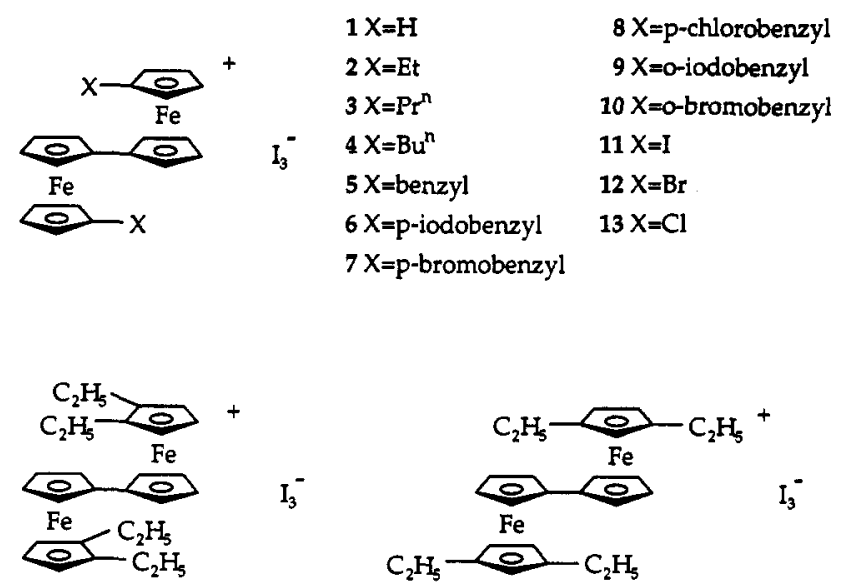

14

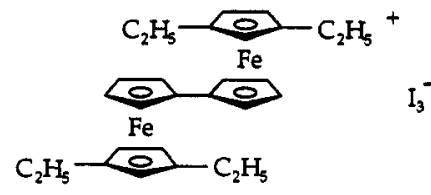

15

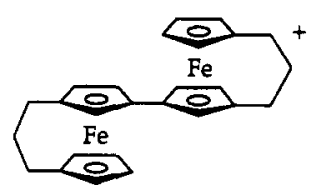

$\mathrm{I}_{3}$

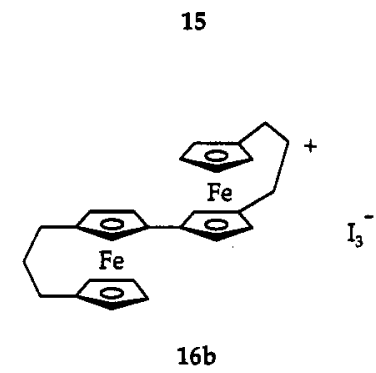

together with no discernible line broadening and eventually to become a single "average-valence" doublet at temperatures of $275,245,275$, and $260 \mathrm{~K}$, respectively. Hendrickson suggested ${ }^{4}$ that the nature of the solid-state environment about a mixed-valence biferrocenium cation can have a dramatic impact on the rate of electron transfer. When there is an onset of dynamics associated with the counterions, or ligands, this will probably influence the rate of intramolecular electron transfer. Very recently, we found that changing the substituent in the benzyl unit in compounds 6-10 leads to dramatic changes in the electron-transfer rate in the solid state. ${ }^{17,18}$ The features in the variable-temperature $\left(77-300 \mathrm{~K}\right.$ ) ${ }^{57} \mathrm{Fe}$ Mössbauer spectra of 6 and 8 include two doublets, which are expected for a mixed-valence cation localized on the time scale of the Mössbauer technique (electron-transfer rates less than $\sim 10^{7} \mathrm{~s}^{-1}$ ). In the case of 7 , at temperatures below $150 \mathrm{~K}$, two doublets are observed in the Mössbauer spectra, and increasing the temperature causes two doublets to become a single average-valence doublet at a temperature of $\sim 200$ $K$. In comparison with compounds $6-8$, a single averagevalence doublet is seen even at $77 \mathrm{~K}$ for 9 and 10 . We suggested ${ }^{18}$ that the interactions between the cation and anion undoubtedly alter the potential energy surface of the mixed-valence molecule and, therefore, the rate of intramolecular electron transfer.

A recent interesting finding is that there is a significant influence on the electron-transfer rate in the mixed-valence biferrocenium salt when the cyclopentadienyl $(\mathrm{Cp})$ rings in each ferrocenyl moiety are tilted from a parallel geometry. ${ }^{21,24-26}$ Such a structural modification of the parallel relation between the two $\mathrm{Cp}$ rings around the $\mathrm{Fe}$

(23) Webb, R. J.; Rheingold, A. L.; Geib, S. J.; Staley, D. L.; Hendrickson, D. N. Angew. Chem., Int. Ed. Engl. 1989, 28, 1388. 427.

(24) Dong, T.-Y.; Lee, T. Y.; Lin, H. M. J. Organomet. Chem. 1992,

(25) Dong, T.-Y.; Chang, C. K.; Huang, C. H.; Wen, Y. S.; Lee, S. L.; Chen, J. A.; Yeh, W. Y.; Yeh, A. J. Chem. Soc., Chem. Commun. 1992, 526.

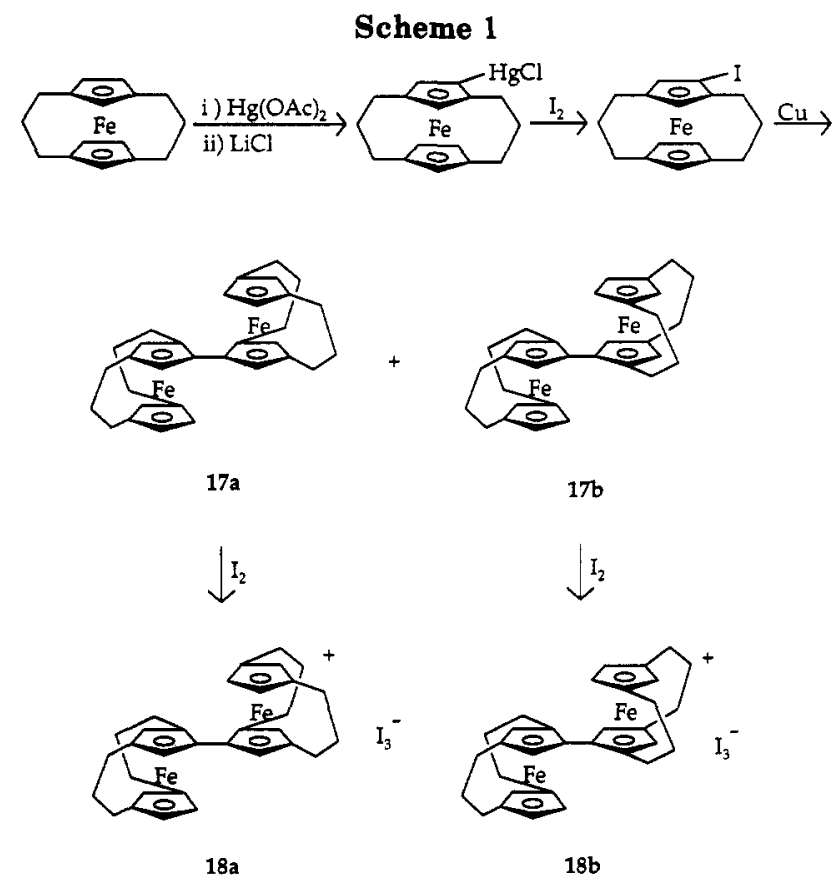

Table 1. Experimental and Crystal Data for the X-ray Structures

\begin{tabular}{|c|c|c|c|c|}
\hline & $17 \mathbf{a}$ & $17 b$ & $18 \mathbf{a}$ & $18 b$ \\
\hline $\begin{array}{l}\text { formula } \\
\text { mw }\end{array}$ & $\begin{array}{l}\mathrm{C}_{32} \mathrm{H}_{34} \mathrm{Fe}_{2} \\
530.31\end{array}$ & $\begin{array}{l}\mathrm{C}_{32} \mathrm{H}_{34} \mathrm{Fe}_{2} \\
530.31\end{array}$ & $\begin{array}{l}\mathrm{C}_{32} \mathrm{H}_{34} \mathrm{Fe}_{2} \mathrm{I}_{3} \\
911.02\end{array}$ & $\begin{array}{l}\mathrm{C}_{32} \mathrm{H}_{34} \mathrm{Fe}_{2} \mathrm{I}_{3} \\
911.02\end{array}$ \\
\hline cryst syst & triclinic & triclinic & monoclinic & monoclinic \\
\hline space group & $P \overline{1}$ & $P \overline{1}$ & $P 2_{1} / c$ & $P 2_{1} / n$ \\
\hline$a, \AA$ & $9.144(1)$ & $9.209(1)$ & $11.363(2)$ & $11.578(1)$ \\
\hline$b, \AA$ & $15.051(2)$ & $24.132(2)$ & $13.666(2)$ & $12.108(2)$ \\
\hline$c, \AA$ & $17.844(4)$ & $24.501(4)$ & $19.753(3)$ & $21.162(3)$ \\
\hline$\alpha, \operatorname{deg}$ & $106.39(3)$ & $116.808(9)$ & & \\
\hline$\beta, \mathrm{deg}$ & $91.73(2)$ & $87.98(1)$ & $104.79(1)$ & $90.379(8)$ \\
\hline$\gamma$, deg & $90.76(2)$ & $91.58(1)$ & & \\
\hline$V, \AA^{3}$ & $2354.3(7)$ & $4856.2(1)$ & $2965.6(7)$ & $2966.5(7)$ \\
\hline$Z$ & 4 & 8 & 4 & 4 \\
\hline$D_{\text {caled, }}, \mathrm{g} \mathrm{cm}^{-3}$ & 1.496 & 1.451 & 2.040 & 2.040 \\
\hline$\mu, \mathrm{mm}^{-1}$ & 1.25 & 1.21 & 4.09 & 4.09 \\
\hline$\lambda, \AA$ & 0.70930 & 0.70930 & 0.70930 & 0.70930 \\
\hline $2 \theta$ limits, deg & 44.9 & 44.8 & 44.9 & 49.8 \\
\hline $\begin{array}{l}\max , \min \\
\text { transmissn } \\
\text { coeff }\end{array}$ & $0.996,0.926$ & $0.999,0.939$ & $0.998,0.601$ & $0.999,0.781$ \\
\hline $\boldsymbol{R}_{F}$ & 0.040 & 0.038 & 0.039 & 0.030 \\
\hline$R_{w F}$ & 0.046 & 0.041 & 0.043 & 0.032 \\
\hline
\end{tabular}

ion would lead to greater metal-ligand interactions as the rings tilt. Compounds 14-16 are of use in understanding the influence of tilted $\mathrm{Cp}$ rings on the rate of electron transfer. ${ }^{25,26}$ Compounds 14 and $1 \dot{5}$ are delocalized on the Mössbauer time scale in the solid state above 195 and 125 $K$, respectively. We suggested ${ }^{26}$ that the difference in electron transfer rates for the series of dialkylbiferrocenium cations is mainly due to the degree of tilting of the $\mathrm{Cp}$ rings from the parallel geometry. Deviations of the $\mathrm{Cp}$ rings from the parallel position were found to correlate quite well with the critical temperature of electronic delocalization-localization in mixed-valence biferrocenium salts. In the case of $\mathbf{1 6}$, there is a significant influence on the electron-transfer rate when the $\mathrm{Cp}$ rings in the ferrocenyl moieties are linked by an interannular trimethylene bridge. ${ }^{21,24}$ The Mössbauer results indicate that compound 16 is delocalized on the Mössbauer time scale above $77 \mathrm{~K}$. Only a single average-valence doublet $\left(\Delta E_{\mathrm{Q}}\right.$

(26) Dong, T.-Y.; Huang, C. H.; Chang, C. K.; Wen, Y. S.; Lee, S. L.; Chen, J. A.; Yeh, W. Y.; Yeh, A. J. Am. Chem. Soc. 1993, 115, 6357. 
Table 2. Atom Coordinates and Thermal Parameters $\left(\AA^{2}\right)$ for 17 a

\begin{tabular}{|c|c|c|c|c|c|c|c|c|c|}
\hline & $x$ & $y$ & $z$ & $B_{\mathrm{iso}}{ }^{a}$ & & $x$ & $y$ & $z$ & $B_{\text {iso }}{ }^{a}$ \\
\hline FelA & $0.76986(8)$ & $0.01167(5)$ & $0.16510(4)$ & $2.66(3)$ & C31A & $0.8280(6)$ & $-0.4011(4)$ & $0.0828(3)$ & $3.9(3)$ \\
\hline FelB & $0.27004(8)$ & $0.49640(5)$ & $0.32340(4)$ & $2.34(3)$ & $\mathrm{C} 32 \mathrm{~A}$ & $0.8405(6)$ & $-0.3686(4)$ & $0.0098(3)$ & $4.1(3)$ \\
\hline $\mathrm{Fe} 2 \mathrm{~A}$ & $0.56661(8)$ & $-0.26275(5)$ & $0.06193(4)$ & $2.26(3)$ & $\mathrm{C} 1 \mathrm{~B}$ & $0.1835(5)$ & $0.6047(3)$ & $0.2900(3)$ & $2.4(2)$ \\
\hline $\mathrm{Fe} 2 \mathrm{~B}$ & $0.05500(8)$ & $0.75840(5)$ & $0.43502(4)$ & $2.66(3)$ & $\mathrm{C} 2 \mathrm{~B}$ & $0.1551(5)$ & $0.5161(3)$ & $0.2325(3)$ & $2.4(2)$ \\
\hline $\mathrm{ClA}$ & $0.7487(6)$ & $-0.1099(3)$ & $0.1924(3)$ & $2.6(2)$ & $\mathrm{C} 3 \mathrm{~B}$ & $0.2937(6)$ & $0.4771(3)$ & $0.2086(3)$ & $2.7(2)$ \\
\hline $\mathrm{C} 2 \mathrm{~A}$ & $0.7514(6)$ & $-0.0312(3)$ & $0.2607(3)$ & $3.1(2)$ & C4B & $0.4067(5)$ & $0.5364(3)$ & $0.2530(3)$ & $2.8(2)$ \\
\hline C3A & $0.8870(6)$ & $0.0172(4)$ & $0.2629(3)$ & $3.6(3)$ & $\mathrm{C} 5 \mathrm{~B}$ & $0.3375(6)$ & $0.6152(3)$ & $0.3020(3)$ & $2.9(3)$ \\
\hline $\mathrm{C} 4 \mathrm{~A}$ & $0.9669(5)$ & $-0.0268(3)$ & $0.1947(3)$ & $3.1(3)$ & C6B & $0.1490(6)$ & $0.4000(3)$ & $0.3502(3)$ & $3.0(2)$ \\
\hline $\mathrm{C} 5 \mathrm{~A}$ & $0.8815(6)$ & $-0.1066(3)$ & $0.1534(3)$ & $2.9(2)$ & $\mathrm{C} 7 \mathrm{~B}$ & $0.2862(6)$ & $0.3639(3)$ & $0.3230(3)$ & $3.0(2)$ \\
\hline C6A & $0.6081(6)$ & $0.1026(4)$ & $0.1777(3)$ & $3.9(3)$ & C8B & $0.3996(6)$ & $0.4190(4)$ & $0.3707(3)$ & $3.5(3)$ \\
\hline C7A & $0.7476(6)$ & $0.1483(4)$ & $0.1804(3)$ & $4.0(3)$ & C9B & $0.3320(6)$ & $0.4865(4)$ & $0.4312(3)$ & $3.5(3)$ \\
\hline C8A & $0.8238(6)$ & $0.1070(3)$ & $0.1109(3)$ & $3.7(3)$ & C10B & $0.1771(6)$ & $0.4745(4)$ & $0.4190(3)$ & $3.4(3)$ \\
\hline $\mathrm{C} 9 \mathrm{~A}$ & $0.7258(6)$ & $0.0392(4)$ & $0.0615(3)$ & $3.8(3)$ & C11B & $0.0708(5)$ & $0.6714(3)$ & $0.3259(3)$ & $2.5(2)$ \\
\hline $\mathrm{ClOA}$ & $0.5943(6)$ & $0.0365(4)$ & $0.1017(3)$ & $3.8(3)$ & C12B & $-0.0583(6)$ & $0.6514(3)$ & $0.3616(3)$ & $3.0(2)$ \\
\hline C11A & $0.6347(5)$ & $-0.1825(3)$ & $0.1697(3)$ & $2.7(2)$ & C13B & $-0.1450(6)$ & $0.7315(4)$ & $0.3842(3)$ & $3.4(3)$ \\
\hline $\mathrm{C} 12 \mathrm{~A}$ & $0.4816(6)$ & $-0.1720(3)$ & $0.1559(3)$ & $3.1(2)$ & C14B & $-0.0706(6)$ & $0.8010(4)$ & $0.3590(3)$ & $3.4(3)$ \\
\hline $\mathrm{C} 13 \mathrm{~A}$ & $0.4095(6)$ & $-0.2594(4)$ & $0.1376(3)$ & $3.2(2)$ & C15B & $0.0649(6)$ & $0.7669(3)$ & $0.3254(3)$ & $3.1(2)$ \\
\hline C14A & $0.5171(6)$ & $-0.3246(3)$ & $0.1435(3)$ & $3.1(2)$ & C16B & $0.0085(6)$ & $0.8095(4)$ & $0.5473(3)$ & $3.6(3)$ \\
\hline C15A & $0.6567(6)$ & $-0.2790(3)$ & $0.1608(3)$ & $2.7(2)$ & $\mathrm{C} 17 \mathrm{~B}$ & $0.0790(6)$ & $0.8780(4)$ & $0.5198(3)$ & $3.6(3)$ \\
\hline $\mathrm{C} 16 \mathrm{~A}$ & $0.7044(6)$ & $-0.3313(4)$ & $-0.0172(3)$ & $3.0(3)$ & $\mathrm{C} 18 \mathrm{~B}$ & $0.2160(6)$ & $0.8455(4)$ & $0.4888(3)$ & $3.8(3)$ \\
\hline C17A & $0.5654(6)$ & $-0.3763(3)$ & $-0.0297(3)$ & $3.0(2)$ & $\mathrm{C} 19 \mathrm{~B}$ & $0.2354(7)$ & $0.7568(4)$ & $0.5027(3)$ & $4.3(3)$ \\
\hline $\mathrm{C} 18 \mathrm{~A}$ & $0.4582(6)$ & $-0.3129(4)$ & $-0.0414(3)$ & $3.4(3)$ & C20B & $0.1082(7)$ & $0.7356(4)$ & $0.5385(3)$ & $4.1(3)$ \\
\hline C19A & $0.5337(6)$ & $-0.2302(4)$ & $-0.0400(3)$ & $3.8(3)$ & C21B & $0.0107(6)$ & $0.4666(3)$ & $0.2082(3)$ & $2.8(2)$ \\
\hline C20A & $0.6838(6)$ & $-0.2412(4)$ & $-0.0258(3)$ & $3.5(3)$ & C22B & $0.0013(6)$ & $0.3706(3)$ & $0.2225(3)$ & $3.3(2)$ \\
\hline $\mathrm{C} 21 \mathrm{~A}$ & $0.6248(7)$ & $0.0022(4)$ & $0.3143(3)$ & $4.2(3)$ & $\mathrm{C} 23 \mathrm{~B}$ & $0.0044(6)$ & $0.3712(4)$ & $0.3087(3)$ & $3.5(3)$ \\
\hline C22A & $0.5772(7)$ & $0.1011(4)$ & $0.3190(4)$ & $5.5(3)$ & C24B & $0.5655(6)$ & $0.5123(4)$ & $0.2549(3)$ & $3.6(3)$ \\
\hline C23A & $0.5086(7)$ & $0.1134(4)$ & $0.2441(4)$ & $5.3(3)$ & $\mathrm{C} 25 \mathrm{~B}$ & $0.5944(6)$ & $0.4187(4)$ & $0.2706(4)$ & $4.8(3)$ \\
\hline C24A & $1.1016(6)$ & $0.0109(4)$ & $0.1675(4)$ & $4.5(3)$ & C26B & $0.5603(6)$ & $0.4139(4)$ & $0.3532(3)$ & $4.4(3)$ \\
\hline $\mathrm{C} 25 \mathrm{~A}$ & $1.0888(6)$ & $0.1098(4)$ & $0.1601(4)$ & $4.5(3)$ & C27B & $-0.2769(7)$ & $0.7452(4)$ & $0.4338(4)$ & $5.1(3)$ \\
\hline C26A & $0.9807(7)$ & $0.1217(4)$ & $0.0974(4)$ & $4.3(3)$ & C28B & $-0.2572(9)$ & $0.807(1)$ & $0.5118(5)$ & $15.6(9)$ \\
\hline C27A & $0.2538(6)$ & $-0.2834(4)$ & $0.1070(3)$ & $4.4(3)$ & C29B & $-0.1471(7)$ & $0.8099(4)$ & $0.5713(3)$ & $4.7(3)$ \\
\hline $\mathrm{C} 28 \mathrm{~A}$ & $0.2415(7)$ & $-0.3596(5)$ & $0.0303(5)$ & $6.9(4)$ & C30B & $0.1819(7)$ & $0.8250(3)$ & $0.3048(3)$ & $3.8(3)$ \\
\hline $\mathrm{C} 29 \mathrm{~A}$ & $0.2960(7)$ & $-0.3266(4)$ & $-0.0423(3)$ & $4.8(3)$ & C31B & $0.2322(7)$ & $0.9096(4)$ & $0.3717(4)$ & $4.6(3)$ \\
\hline $\mathrm{C} 30 \mathrm{~A}$ & $0.8013(6)$ & $-0.3262(4)$ & $0.1591(3)$ & $3.6(3)$ & C32B & $0.3111(7)$ & $0.8901(4)$ & $0.4413(4)$ & $4.9(3)$ \\
\hline
\end{tabular}

${ }^{a} B_{\text {iso }}$ is the mean of the principal axes of the thermal ellipsoid.

$=1.614 \mathrm{~mm} \mathrm{~s}^{-1}$ ) is observed from 77 to $300 \mathrm{~K}$. In our previous papers, ${ }^{21,24}$ we proposed that such a structural modification would lead to increased metal-ligand interactions as the rings tilt. However, there is still one important question that remains. At temperatures below $5 \mathrm{~K}$, compound 16 shows two doublets in the ${ }^{57} \mathrm{Fe}$ Mössbauer spectra, one with a quadrupole splitting $\left(\Delta E_{\mathrm{Q}}\right)$ of $\sim 1.36 \mathrm{~mm} \mathrm{~s}^{-1}$ and the other with $\Delta E_{Q} \sim 1.88 \mathrm{~mm} \mathrm{~s}^{-1} .27$ Two possible explanations for this ${ }^{57} \mathrm{Fe}$ Mössbauer spectrum may be taken into account. First, the Mössbauer results indicate that the valence state of iron atoms in compound 16 is localized on the Mössbauer time scale $\left(10^{7} \mathrm{~s}^{-1}\right)$ in the solid state at $4.2 \mathrm{~K}$. A second possibility is that the Mössbauer sample contained both constitutional isomers $16 \mathbf{a}, \mathbf{b}$ in a $1: 1$ ratio. Unfortunately, a suitable crystal of 16 for X-ray determination cannot be obtained. To study the effects of interannular bridges, we have prepared the two new constitutional isomers 18a,b (Scheme 1) and determined the crystal structures of $17 \mathbf{a}, \mathbf{b}$ and $18 \mathbf{a}, \mathbf{b}$. We now show that there is a dramatic difference in electron-transfer rates between 16 and 18. Furthermore, the unusual physical properties of 18 are explained in terms of structural characteristics.

\section{Experimental Section}

General Information. All manipulations involving airsensitive materials were carried out by using standard Schlenk techniques under an atmosphere of $\mathrm{N}_{2}$. Chromatography was performed on neutral alumina (activity II), with hexane $/ \mathrm{CH}_{2} \mathrm{Cl}_{2}$ as eluent. Solvents were purified as follows: benzene, hexane, and ether distilled from $\mathrm{Na}$ / benzophenone; $\mathrm{CH}_{2} \mathrm{Cl}_{2}$, distilled from

(27) Unpublished results
$\mathrm{P}_{2} \mathrm{O}_{5} . \quad 1,1^{\prime}: 3,3^{\prime}$-Bis(propane-1,3-diyl)ferrocene was prepared according to the literature procedure ${ }^{28}$ and identified by its melting point, NMR, and mass spectrum.

Mercuration of $1,1^{\prime}: 3,3^{\prime}$-Bis (propane-1,3-diyl)ferrocene. $\mathrm{ACH}_{3} \mathrm{OH}(75 \mathrm{~mL})$ solution of mercuric acetate $(2.6 \mathrm{~g}, 8.16 \mathrm{mmol})$ was added dropwise to a hot solution of $1,1^{\prime}: 3,3^{\prime}$-bis(propane1,3-diyl)ferrocene ( $3.5 \mathrm{~g}, 13.15 \mathrm{mmol})$ dissolved in $80 \mathrm{~mL}$ of absolute $\mathrm{CH}_{3} \mathrm{OH}$ and $50 \mathrm{~mL}$ of dry ether. The mixture was refluxed for $18 \mathrm{~h}$, and then $\mathrm{LiCl}(0.5 \mathrm{~g})$ in $65 \mathrm{~mL}$ of hot $\mathrm{CH}_{3} \mathrm{OH}$ was added dropwise. A yellow precipitate was formed immediately out of an orange solution. The solvent was removed under vacuum. The resulting yellow powder was put into a Soxhlet extractor and extracted with hexane to yield the starting material. Further extraction with $\mathrm{CH}_{2} \mathrm{Cl}_{2}$ yielded $1.36 \mathrm{~g}(33 \%)$ of a yellow solid. The crude product was used without further purification.

Iodination of Mercurated 1,1':3,3'-Bis(propane-1,3-diyl)ferrocene. $N$-Iodosuccinimide (NIS; $0.78 \mathrm{~g}, 3.5 \mathrm{mmol}$ ) in $75 \mathrm{~mL}$ of $\mathrm{CH}_{2} \mathrm{Cl}_{2}$ was added dropwise at $0^{\circ} \mathrm{C}$ to $1.36 \mathrm{~g}(2.71 \mathrm{mmol})$ of mercurated 1,1':3,3'-bis(propane-1,3-diyl)ferrocene. The reaction mixture was warmed to room temperature and was stirred overnight. To this was added $50 \mathrm{~mL}$ of $10 \%$ aqueous $\mathrm{Na}_{2} \mathrm{~S}_{2} \mathrm{O}_{3}$ followed by $50 \mathrm{~mL}$ of $10 \%$ aqueous $\mathrm{NaHCO}_{3}$. The organic layers were separated, and the aqueous portion was extracted with 3 $\times 50 \mathrm{~mL}$ portions of $\mathrm{CH}_{2} \mathrm{Cl}_{2}$. The combined organic layer was washed twice with $100 \mathrm{~mL}$ of $10 \%$ aqueous $\mathrm{NaHCO}_{3}$ and twice with $100 \mathrm{~mL}$ of $\mathrm{H}_{2} \mathrm{O}$ and dried over $\mathrm{MgSO}_{4}$. The solvent was removed under reduced pressure. The red oily residue was chromatographed, with hexane as eluent. The first band was the desired compound $(\sim 0.5 \mathrm{~g}, 50 \%)$. The properties of 4-iodo1,1':3,3'-bis(propane-1,3-diyl)ferrocene are as follows: ${ }^{1} \mathrm{H}$ NMR ( $\left.\mathrm{CDCl}_{3}, \mathrm{ppm}\right) 3.5-4.5(5 \mathrm{H}, \mathrm{m}$, ring protons), 1.5-2.5 $(12 \mathrm{H}, \mathrm{m}$, methylene protons); mass spectrum $\mathbf{M}^{+}$at $m / z 392$.

(28) Crawford, W.; Turbitt, T. D.; Watts, W. E. J. Organomet. Chem. $1976,105,341$. 
Table 3. Selected Bond Distances $(\AA)$ and Bond Angles (deg)

\begin{tabular}{|c|c|c|c|c|}
\hline & \multicolumn{2}{|c|}{$17 \mathbf{a}$} & \multirow[b]{2}{*}{$18 a$} & \multirow[b]{2}{*}{$18 \mathrm{~b}$} \\
\hline & A & B & & \\
\hline $\mathrm{I} 1-\mathrm{I} 2$ & & & $2.905(2)$ & $2.9393(6)$ \\
\hline $\mathrm{I} 1-\mathrm{I} 3$ & & & $2.908(3)$ & $2.9058(7)$ \\
\hline $\mathrm{Fe} 1-\mathrm{C} 1$ & $2.030(5)$ & $2.047(5)$ & $2.042(9)$ & $2.136(5)$ \\
\hline $\mathrm{Fe} 1-\mathrm{C} 2$ & $1.997(5)$ & $2.001(4)$ & $2.02(1)$ & $2.031(5)$ \\
\hline $\mathrm{Fe} 1-\mathrm{C} 3$ & $2.000(5)$ & $2.001(5)$ & $2.03(1)$ & $2.019(5)$ \\
\hline $\mathrm{Fel}-\mathrm{C} 4$ & $2.000(5)$ & $2.001(5)$ & $2.01(1)$ & $2.032(5)$ \\
\hline $\mathrm{Fel}-\mathrm{C} 5$ & $2.027(5)$ & $2.023(5)$ & $2.02(1)$ & $2.088(5)$ \\
\hline $\mathrm{Fe} 1-\mathrm{C} 6$ & $2.002(5)$ & $1.985(5)$ & $2.02(1)$ & $2.036(5)$ \\
\hline $\mathrm{Fel}-\mathrm{C} 7$ & $2.011(5)$ & $2.000(5)$ & $2.00(1)$ & $2.014(5)$ \\
\hline $\mathrm{Fel}-\mathrm{C} 8$ & $2.007(5)$ & $2.001(5)$ & $2.00(1)$ & $2.042(5)$ \\
\hline $\mathrm{Fe} 1-\mathrm{C} 9$ & $2.035(5)$ & $2.035(5)$ & $2.03(1)$ & $2.099(5)$ \\
\hline $\mathrm{Fe} 1-\mathrm{C} 10$ & $2.035(5)$ & $2.034(5)$ & $2.02(1)$ & $2.089(5)$ \\
\hline $\mathrm{Fe} 2-\mathrm{C} 11$ & $2.035(5)$ & $2.028(5)$ & $2.099(9)$ & $2.031(5)$ \\
\hline $\mathrm{Fe} 2-\mathrm{C} 12$ & $2.024(5)$ & $2.016(5)$ & $2.06(1)$ & $2.023(5)$ \\
\hline $\mathrm{Fe} 2-\mathrm{C} 13$ & $1.992(5)$ & $2.001(5)$ & $2.02(1)$ & $2.006(5)$ \\
\hline $\mathrm{Fe} 2-\mathrm{C} 14$ & $1.997(5)$ & $1.997(5)$ & $2.02(1)$ & $2.006(5)$ \\
\hline $\mathrm{Fe} 2-\mathrm{C} 15$ & $2.003(5)$ & $2.001(5)$ & $2.05(1)$ & $2.011(5)$ \\
\hline $\mathrm{Fe} 2-\mathrm{C} 16$ & $1.991(5)$ & $1.994(5)$ & $2.05(1)$ & $2.011(5)$ \\
\hline $\mathrm{Fe} 2-\mathrm{C} 17$ & $2.004(5)$ & $2.002(5)$ & $2.07(1)$ & $2.012(5)$ \\
\hline $\mathrm{Fe} 2-\mathrm{C} 18$ & $1.991(5)$ & $1.991(5)$ & $2.08(1)$ & $2.008(5)$ \\
\hline $\mathrm{Fe} 2-\mathrm{C} 19$ & $2.019(6)$ & $2.019(6)$ & $2.06(1)$ & $2.031(5)$ \\
\hline $\mathrm{Fe} 2-\mathrm{C} 20$ & $2.018(5)$ & $2.018(5)$ & $2.05(1)$ & $2.025(5)$ \\
\hline $\mathrm{Cl}-\mathrm{C} 2$ & $1.439(7)$ & $1.449(6)$ & $1.46(2)$ & $1.449(7)$ \\
\hline $\mathrm{Cl}-\mathrm{Cs}$ & $1.424(7)$ & $1.418(7)$ & $1.44(2)$ & $1.436(7)$ \\
\hline $\mathrm{Cl}-\mathrm{C} 11$ & $1.462(7)$ & $1.478(7)$ & $1.44(1)$ & $1.455(7)$ \\
\hline $\mathrm{C} 2-\mathrm{C} 3$ & $1.424(8)$ & $1.428(7)$ & $1.49(2)$ & $1.422(7)$ \\
\hline $\mathrm{C} 3-\mathrm{C} 4$ & $1.437(8)$ & $1.421(7)$ & $1.44(2)$ & $1.424(8)$ \\
\hline $\mathrm{C} 4-\mathrm{C} 5$ & $1.428(7)$ & $1.427(7)$ & $1.43(2)$ & $1.427(7)$ \\
\hline C6-C7 & $1.435(8)$ & $1.418(7)$ & $1.44(2)$ & $1.422(7)$ \\
\hline C6-C10 & $1.438(9)$ & $1.424(7)$ & $1.45(2)$ & $1.425(8)$ \\
\hline $\mathrm{C} 7-\mathrm{C} 8$ & $1.427(8)$ & $1.419(8)$ & $1.41(2)$ & $1.421(8)$ \\
\hline $\mathrm{C} 8-\mathrm{Cg}$ & $1.429(8)$ & $1.420(8)$ & $1.43(2)$ & $1.440(8)$ \\
\hline C9-C10 & $1.425(9)$ & $1.427(8)$ & $1.38(2)$ & $1.404(8)$ \\
\hline $\mathrm{C} 11-\mathrm{C} 12$ & $1.433(7)$ & $1.428(7)$ & $1.46(1)$ & $1.439(7)$ \\
\hline $\mathrm{C} 11-\mathrm{C} 15$ & $1.433(7)$ & $1.441(7)$ & $1.44(1)$ & $1.455(7)$ \\
\hline $\mathrm{C} 12-\mathrm{C} 13$ & $1.411(7)$ & $1.420(7)$ & $1.42(1)$ & $1.421(8)$ \\
\hline $\mathrm{Cl3}-\mathrm{Cl} 4$ & $1.422(8)$ & $1.422(8)$ & $1.41(2)$ & $1.434(8)$ \\
\hline $\mathrm{Cl} 4-\mathrm{Cl} 5$ & $1.425(7)$ & $1.431(8)$ & $1.44(2)$ & $1.428(7)$ \\
\hline $\mathrm{C} 16-\mathrm{C} 17$ & $1.413(7)$ & $1.416(8)$ & $1.44(2)$ & $1.429(9)$ \\
\hline $\mathrm{C} 16-\mathrm{C} 20$ & $1.422(7)$ & $1.424(8)$ & $1.45(2)$ & $1.436(9)$ \\
\hline $\mathrm{C} 17-\mathrm{C} 18$ & $1.428(7)$ & $1.421(8)$ & $1.43(2)$ & $1.424(8)$ \\
\hline $\mathrm{C} 18-\mathrm{C} 19$ & $1.409(8)$ & $1.438(8)$ & $1.42(2)$ & $1.425(9)$ \\
\hline $\mathrm{C} 19-\mathrm{C} 20$ & $1.408(8)$ & $1.419(9)$ & $1.39(2)$ & $1.423(9)$ \\
\hline $\mathrm{I} 2-\mathrm{I} 1-\mathrm{I} 3$ & & & $177.26(4)$ & $175.65(2)$ \\
\hline $\mathrm{C} 2-\mathrm{C} 1-\mathrm{C} 5$ & $107.2(4)$ & $107.0(4)$ & $109.1(9)$ & $106.5(4)$ \\
\hline $\mathrm{C} 2-\mathrm{C} 1-\mathrm{C} 11$ & $126.3(4)$ & $125.3(4)$ & $125(1)$ & $128.4(4)$ \\
\hline $\mathrm{C} 5-\mathrm{C} 1-\mathrm{C} 11$ & $126.4(4)$ & $127.7(4)$ & $125.5(9)$ & $125.1(4)$ \\
\hline $\mathrm{Cl}-\mathrm{C} 2-\mathrm{C}_{3}$ & $107.4(5)$ & $107.3(4)$ & $105(1)$ & $107.3(4)$ \\
\hline $\mathrm{C} 2-\mathrm{C} 3-\mathrm{C} 4$ & $109.4(4)$ & $109.1(4)$ & $109(1)$ & $109.9(4)$ \\
\hline $\mathrm{C} 3-\mathrm{C} 4-\mathrm{C} 5$ & $106.0(4)$ & $106.9(4)$ & $108(1)$ & $106.3(4)$ \\
\hline $\mathrm{Cl}-\mathrm{C} 5-\mathrm{C} 4$ & $109.8(4)$ & $109.6(4)$ & $109(1)$ & $109.7(4)$ \\
\hline $\mathrm{C} 7-\mathrm{C} 6-\mathrm{C} 10$ & $105.9(5)$ & $107.3(5)$ & $103(1)$ & $105.2(5)$ \\
\hline $\mathrm{C} 6-\mathrm{C} 7-\mathrm{C} 8$ & $110.0(5)$ & $109.1(4)$ & $110(1)$ & $110.8(5)$ \\
\hline $\mathrm{C} 7-\mathrm{C} 8-\mathrm{C} 9$ & $106.5(5)$ & $107.3(5)$ & $108(1)$ & $105.7(5)$ \\
\hline $\mathrm{C} 8-\mathrm{C} 9-\mathrm{C} 10$ & $108.7(5)$ & $108.2(5)$ & $107(1)$ & $108.2(5)$ \\
\hline $\mathrm{C} 6-\mathrm{C} 10-\mathrm{C} 9$ & $108.7(5)$ & $108.0(5)$ & $111(1)$ & $110.0(5)$ \\
\hline $\mathrm{Cl}-\mathrm{C} 11-\mathrm{C} 12$ & $127.9(4)$ & $126.3(4)$ & $125.4(9)$ & $128.0(4)$ \\
\hline $\mathrm{C} 1-\mathrm{C} 11-\mathrm{C} 15$ & $125.2(4)$ & $126.2(4)$ & $126.2(9)$ & $124.3(4)$ \\
\hline $\mathrm{C}_{12}-\mathrm{C}_{11}-\mathrm{C}_{15}$ & $106.9(4)$ & $107.3(4)$ & $107.9(8)$ & $107.6(4)$ \\
\hline $\mathrm{C} 11-\mathrm{C} 12-\mathrm{C}_{13}$ & $109.6(4)$ & $110.0(4)$ & $108.0(8)$ & $109.0(4)$ \\
\hline $\mathrm{C} 12-\mathrm{C} 13-\mathrm{C} 14$ & $106.7(4)$ & $106.0(5)$ & $107.7(9)$ & $106.9(4)$ \\
\hline $\mathrm{C} 13-\mathrm{C} 14-\mathrm{C} 15$ & $109.4(4)$ & $110.3(4)$ & $110.3(9)$ & $110.0(4)$ \\
\hline $\mathrm{C} 11-\mathrm{C} 15-\mathrm{C} 14$ & $107.3(4)$ & $106.4(5)$ & $105.9(9)$ & $106.4(4)$ \\
\hline $\mathrm{C} 17-\mathrm{C} 16-\mathrm{C} 20$ & $106.6(4)$ & $106.7(5)$ & $106(1)$ & $105.0(5)$ \\
\hline $\mathrm{C} 16-\mathrm{C} 17-\mathrm{C} 18$ & $109.1(4)$ & $109.8(5)$ & $110(1)$ & $110.8(5)$ \\
\hline $\mathrm{C} 17-\mathrm{C} 18-\mathrm{C} 19$ & $106.9(5)$ & $106.6(5)$ & $106(1)$ & $106.4(5)$ \\
\hline $\mathrm{C} 18-\mathrm{C} 19-\mathrm{C} 20$ & $108.6(5)$ & $107.8(5)$ & $111(1)$ & $108.3(5)$ \\
\hline $\mathrm{C} 16-\mathrm{C} 20-\mathrm{C} 19$ & $108.7(5)$ & $109.0(5)$ & $108(1)$ & $109.4(5)$ \\
\hline
\end{tabular}

Neutral Compounds $17 a, b$. Compounds $17 \mathbf{a}, \mathbf{b}$ were prepared by the Ullman coupling procedure as shown in Scheme 1 . A mixture of 4-iodo-1,1':3,3'-bis (propane-1,3-diyl)ferrocene $(0.5 \mathrm{~g})$ and activated copper $(2 \mathrm{~g})$ was heated under $\mathrm{N}_{2}$ at $110-120^{\circ} \mathrm{C}$ for $24 \mathrm{~h}$. After it was cooled to room temperature, the reaction mixture was repeatedly extracted with $\mathrm{CH}_{2} \mathrm{Cl}_{2}$ until the $\mathrm{CH}_{2} \mathrm{Cl}_{2}$ extracts appeared colorless. The extracts were evaporated and chromatographed on neutral $\mathrm{Al}_{2} \mathrm{O}_{3}$. The first band eluted with hexane yielded the starting material. Continued elution with hexane afforded the desired compounds $17 \mathrm{a}(0.1 \mathrm{~g}$, second band) and $17 \mathrm{~b}(0.1 \mathrm{~g}$, third band $)$. Compounds $17 \mathrm{a}, \mathrm{b}$ were recrystallized from benzene/hexane. 17a: ${ }^{1} \mathrm{H} \mathrm{NMR}\left(\mathrm{CDCl}_{3}, \mathrm{ppm}\right) 4.12(\mathrm{~s}, 2 \mathrm{H}$, Cp), 4.07 (s, 2H, Cp), 3.79 (s, 2H, Cp), 3.73 (s, 2H, Cp), 3.59 (s, $2 \mathrm{H}, \mathrm{Cp}), 2.2-1.4\left(\mathrm{~m}, 24 \mathrm{H},-\mathrm{CH}_{2}-\right)$; $\mathrm{mp} 242-243^{\circ} \mathrm{C}$; mass spectrum $\mathrm{M}^{+}$at $m / z$ 530. 17b: ${ }^{1} \mathrm{H}$ NMR (CDCl, $\left.\mathrm{ppm}\right) 3.99$ (s, $\left.4 \mathrm{H}, \mathrm{Cp}\right)$, 3.82 (s, 2H, Cp), 3.73 (s, 2H, Cp), 3.47 (s, 2H, Cp), 2.3-1.4 (m, $24 \mathrm{H},-\mathrm{CH}_{2}-$ ); $m p \quad 192-193^{\circ} \mathrm{C}$; mass spectrum $\mathrm{M}^{+}$at $m / z 530$.

Mixed-Valence Compounds $18 a, b$. A crystalline sample of 18a was prepared by adding a benzene/hexane (1:1) solution containing a stoichiometric amount of iodine to a benzene/hexane (1:1) solution of the corresponding biferrocene at $0^{\circ} \mathrm{C}$. The resulting dark green crystals were filtered and washed repeatedly with cold hexane. The same method described for the preparation of 18a was used to synthesize 18b, except dry ether was used instead of benzene/hexane. Anal. Calcd for $18 \mathrm{a}\left(\mathrm{C}_{32} \mathrm{H}_{34} \mathrm{Fe}_{2} \mathrm{I}_{3}\right)$ : $\mathrm{C}, 42.19 ; \mathrm{H}, 3.76$. Found: $\mathrm{C}, 42.03 ; \mathrm{H}, 3.80$. Calcd for $18 \mathrm{~b}$ $\left(\mathrm{C}_{32} \mathrm{H}_{34} \mathrm{Fe}_{2} \mathrm{I}_{3}\right): \mathrm{C}, 42.19 ; \mathrm{H}, 3.76$. Found: $\mathrm{C}, 42.19 ; \mathrm{H}, 3.44$.

Physical Methods. ${ }^{57} \mathrm{Fe}$ Mössbauer measurements were made on a constant-velocity instrument which has been previously described. ${ }^{18}$ The absolute temperature accuracy is estimated to be $\pm 5 \mathrm{~K}$, while the relative precision is $\pm 0.5 \mathrm{~K}$. Computer fittings of the ${ }^{57} \mathrm{Fe}$ Mössbauer data to Lorentzian lines were carried out with a modified version of a previously reported program. ${ }^{29}$ Velocity calibrations were made using a $99.99 \%$ pure $10-\mu \mathrm{m}$ iron foil. Typical line widths for all three pairs of iron lines fell in the range $0.24-0.27 \mathrm{~mm} / \mathrm{s}$. Isomer shifts are reported relative to iron foil at $300 \mathrm{~K}$ but are uncorrected for temperature-dependent, second-order Doppler effects. It should be noted that the isomer shifts illustrated in the figures are plotted as obtained experimentally. Tabulated data are provided.

${ }^{1} \mathrm{H}$ NMR spectra were recorded on a Bruker AMX500 spectrometer. Mass spectra were obtained with a VG250-70S system. The near-IR spectra were recorded from 2300 to $900 \mathrm{~nm}$ in $\mathrm{CH}_{2} \mathrm{Cl}_{2}$ by using $1.0-\mathrm{cm}$ quartz cells with a Perkin-Elmer Lambda 9 spectrophotometer.

Electrochemical measurements were carried out with a BAS $100 \mathrm{~B}$ system. Cyclic voltammetry was performed with a stationary glassy-carbon working electrode, which was cleaned after each run. These experiments were carried out with $1 \times 10^{-3} \mathrm{M}$ solutions of biferrocene in dry $\mathrm{CH}_{2} \mathrm{Cl}_{2} / \mathrm{CH}_{3} \mathrm{CN}$ (1:1) containing $0.1 \mathrm{M}$ of $\left(n-\mathrm{C}_{4} \mathrm{H}_{9}\right)_{4} \mathrm{NPF}_{6}$ as supporting electrolyte. The potentials quoted in this work are relative to a $\mathrm{Ag} / \mathrm{AgCl}$ electrode at $25^{\circ} \mathrm{C}$. Under these conditions, ferrocene shows a reversible one-electronoxidation wave $\left(E_{1 / 2}=0.37 \mathrm{~V}\right)$.

The single-crystal X-ray determinations of compounds $17 \mathbf{a}, \mathbf{b}$ and $18 \mathbf{a}, \mathbf{b}$ were carried out on an Enraf-Nonius CAD4 diffractometer at $298 \mathrm{~K}$. Absorption corrections were made with empirical $\psi$ rotation for all four compounds. The X-ray crystal data are summarized in Table 1 . Complete tables of bond distances and angles and of thermal parameters for these compounds are given as supplementary material.

Structure Determination of $17 \mathrm{a}$. An orange crystal $(0.29$ $\times 0.28 \times 0.34 \mathrm{~mm}$ ), which was grown by slow evaporation from a hexane solution, was used for data collection at $298 \mathrm{~K}$. Cell dimensions were obtained from 20 reflections with $14.95^{\circ}<2 \theta$ $<29.41^{\circ}$. The $\theta-2 \theta$ scan technique was used to record the intensities for all reflections for which $1^{\circ}<2 \theta<44.9^{\circ}$. Of the 6139 unique reflections, there were 4458 reflections with $F_{0}>$ $2.0 \sigma\left(F_{0}{ }^{2}\right)$, where $\sigma\left(F_{0}{ }^{2}\right)$ values were estimated from counting statistics. These data were used in the final refinement of the structural parameters.

A three-dimensional Patterson synthesis was used to determine the heavy-atom positions, which phased the data sufficiently well to permit location of the remaining non-hydrogen atoms from Fourier synthesis. All non-hydrogen atoms were refined

(29) Lee, J. F.; Lee, M. D.; Tseng, P. K. Chemistry 1987, 45, 50. 
Table 4. Atom Coordinates and Thermal Parameters $\left(\AA^{2}\right)$ for $17 \mathrm{~b}$

\begin{tabular}{|c|c|c|c|c|c|c|c|c|c|}
\hline & $x$ & $y$ & $z$ & $B_{\text {iso }}$ & & $x$ & $y$ & $z$ & $B_{\text {iso }}$ \\
\hline $\mathrm{FelA}$ & $0.3252(1)$ & $0.65726(5)$ & $0.26522(6)$ & $2.91(6)$ & FelC & $0.0645(1)$ & $0.16076(5)$ & $0.97554(6)$ & $3.87(7)$ \\
\hline $\mathrm{Fe} 2 \mathrm{~A}$ & $0.2686(1)$ & $0.87082(5)$ & $0.28248(5)$ & $3.45(7)$ & $\mathrm{Fe} 2 \mathrm{C}$ & $0.3337(1)$ & $0.36133(5)$ & $1.02230(5)$ & $3.35(7)$ \\
\hline $\mathrm{C} 1 \mathrm{~A}$ & $0.3714(8)$ & $0.7351(3)$ & $0.2543(3)$ & $2.6(4)^{\prime}$ & $\mathrm{ClC}$ & $0.0991(8)$ & $0.2528(3)$ & $0.9996(3)$ & $3.2(4)$ \\
\hline $\mathrm{C} 2 \mathrm{~A}$ & $0.2693(8)$ & $0.6936(3)$ & $0.2100(3)$ & $2.9(4)$ & $\mathrm{C} 2 \mathrm{C}$ & $0.0977(9)$ & $0.2391(4)$ & $1.0519(3)$ & $3.7(5)$ \\
\hline $\mathrm{C} 3 \mathrm{~A}$ & $0.3423(9)$ & $0.6364(3)$ & $0.1765(3)$ & $3.5(5)$ & $\mathrm{C} 3 \mathrm{C}$ & $-0.0424(9)$ & $0.2131(4)$ & $1.0533(4)$ & $4.2(5)$ \\
\hline $\mathrm{C} 4 \mathrm{~A}$ & $0.4820(9)$ & $0.6407(3)$ & $0.2105(3)$ & $3.3(4)$ & $\mathrm{C} 4 \mathrm{C}$ & $-0.1199(9)$ & $0.2060(4)$ & $1.0026(4)$ & $4.9(5)$ \\
\hline $\mathrm{C} 5 \mathrm{~A}$ & $0.4990(8)$ & $0.7016(3)$ & $0.2484(3)$ & $2.8(4)$ & $\mathrm{CsC}$ & $-0.0361(9)$ & $0.2317(4)$ & $0.9710(4)$ & $4.4(5)$ \\
\hline $\mathrm{C} 6 \mathrm{~A}$ & $0.1352(9)$ & $0.6377(4)$ & $0.2908(4)$ & $3.9(5)$ & $\mathrm{C} 6 \mathrm{C}$ & $0.2165(9)$ & $0.1051(4)$ & $0.9791(4)$ & $4.9(6)$ \\
\hline $\mathrm{C} 6 \mathrm{~A}$ & $0.2130(9)$ & $0.5825(3)$ & $0.2574(3)$ & $3.8(5)$ & $\mathrm{C} 7 \mathrm{C}$ & $0.073(1)$ & $0.0816(4)$ & $0.9815(4)$ & $5.1(6)$ \\
\hline $\mathrm{C} 8 \mathrm{~A}$ & $0.3463(9)$ & $0.5859(3)$ & $0.2854(3)$ & $3.5(5)$ & $\mathrm{C} 8 \mathrm{C}$ & $-0.004(1)$ & $0.0728(4)$ & $0.9298(5)$ & $5.9(6)$ \\
\hline $\mathrm{C} 9 \mathrm{~A}$ & $0.3443(9)$ & $0.6422(4)$ & $0.3407(3)$ & $3.6(5)$ & $\mathrm{C} 9 \mathrm{C}$ & $0.094(1)$ & $0.0872(4)$ & $0.8916(4)$ & $6.4(6)$ \\
\hline $\mathrm{C} 10 \mathrm{~A}$ & $0.2143(9)$ & $0.6738(4)$ & $0.3443(3)$ & $3.9(5)$ & $\mathrm{C} 10 \mathrm{C}$ & $0.224(1)$ & $0.1072(4)$ & $0.9223(4)$ & $5.7(6)$ \\
\hline $\mathrm{C} 11 \mathrm{~A}$ & $0.3487(8)$ & $0.8016(3)$ & $0.2987(3)$ & $3.0(5)$ & $\mathrm{C} 11 \mathrm{C}$ & $0.2158(9)$ & $0.2801(3)$ & $0.9772(3)$ & $3.4(5)$ \\
\hline $\mathrm{C} 12 \mathrm{~A}$ & $0.4538(9)$ & $0.8499(3)$ & $0.3086(3)$ & $3.3(5)$ & $\mathrm{C} 12 \mathrm{C}$ & $0.195(1)$ & $0.3252(4)$ & $0.9537(4)$ & $4.5(5)$ \\
\hline $\mathrm{C} 13 \mathrm{~A}$ & $0.3918(9)$ & $0.9055(3)$ & $0.3561(4)$ & $3.5(5)$ & $\mathrm{C} 13 \mathrm{C}$ & $0.328(1)$ & $0.3377(4)$ & $0.9330(4)$ & $4.7(6)$ \\
\hline $\mathrm{C} 14 \mathrm{~A}$ & $0.2500(9)$ & $0.8933(3)$ & $0.3714(3)$ & $3.3(4)$ & $\mathrm{C} 14 \mathrm{C}$ & $0.4375(9)$ & $0.3053(4)$ & $0.9450(4)$ & $4.3(5)$ \\
\hline $\mathrm{C} 15 \mathrm{~A}$ & $0.2236(8)$ & $0.8281(3)$ & $0.3361(3)$ & $3.0(4)$ & $\mathrm{C} 15 \mathrm{C}$ & $0.367(1)$ & $0.2693(3)$ & $0.9708(4)$ & $4.2(5)$ \\
\hline $\mathrm{C} 16 \mathrm{~A}$ & $0.337(1)$ & $0.8892(4)$ & $0.2141(4)$ & $5.0(6)$ & $\mathrm{C} 16 \mathrm{C}$ & $0.243(1)$ & $0.4427(4)$ & $1.0784(4)$ & $4.4(5)$ \\
\hline $\mathrm{C} 17 \mathrm{~A}$ & $0.274(1)$ & $0.9423(4)$ & $0.2613(4)$ & $4.8(6)$ & $\mathrm{Cl}$ C & $0.379(1)$ & $0.4521(4)$ & $1.0541(4)$ & $4.3(5)$ \\
\hline C $18 A$ & $0.127(1)$ & $0.9290(4)$ & $0.2737(4)$ & $5.3(6)$ & $\mathrm{C} 18 \mathrm{C}$ & $0.488(1)$ & $0.4220(4)$ & $1.0689(4)$ & $4.5(5)$ \\
\hline C19A & $0.099(1)$ & $0.8668(4)$ & $0.2303(4)$ & $5.7(6)$ & $\mathrm{C} 19 \mathrm{C}$ & $0.421(1)$ & $0.3970(4)$ & $1.1059(4)$ & $4.5(5)$ \\
\hline $\mathrm{C} 20 \mathrm{~A}$ & $0.227(1)$ & $0.8423(4)$ & $0.1933(4)$ & $5.3(6)$ & $\mathrm{C} 20 \mathrm{C}$ & $0.272(1)$ & $0.4089(4)$ & $1.1114(4)$ & $4.5(5)$ \\
\hline $\mathrm{C} 21 \mathrm{~A}$ & $0.1125(9)$ & $0.7040(3)$ & $0.2038(4)$ & $3.9(5)$ & $\mathrm{C} 21 \mathrm{C}$ & $0.224(1)$ & $0.2449(4)$ & $1.0919(4)$ & $4.6(5)$ \\
\hline $\mathrm{C} 22 \mathrm{~A}$ & $0.006(1)$ & $0.6570(4)$ & $0.2019(4)$ & $5.2(6)$ & $\mathrm{C} 22 \mathrm{C}$ & $0.261(1)$ & $0.1843(5)$ & $1.0933(4)$ & $6.4(7)$ \\
\hline $\mathrm{C} 23 \mathrm{~A}$ & $-0.005(1)$ & $0.6566(4)$ & $0.2727(4)$ & $5.1(6)$ & $\mathrm{C} 23 \mathrm{C}$ & $0.319(1)$ & $0.1328(5)$ & $1.0317(6)$ & $7.6(9)$ \\
\hline $\mathrm{C} 24 \mathrm{~A}$ & $0.582(1)$ & $0.5859(4)$ & $0.1852(4)$ & $4.8(5)$ & $\mathrm{C} 24 \mathrm{C}$ & $-0.262(1)$ & $0.1709(4)$ & $0.9831(5)$ & $7.3(7)$ \\
\hline C25A & $0.600(1)$ & $0.5678(4)$ & $0.2368(4)$ & $5.7(6)$ & $\mathrm{C} 25 \mathrm{C}$ & $-0.259(1)$ & $0.1061(6)$ & $0.9491(7)$ & $12(1)$ \\
\hline $\mathrm{C} 26 \mathrm{~A}$ & $0.467(1)$ & $0.5413(4)$ & $0.2565(4)$ & $4.7(6)$ & $\mathrm{C} 26 \mathrm{C}$ & $-0.160(1)$ & $0.0590(4)$ & $0.9204(5)$ & $8.2(8)$ \\
\hline $\mathrm{C} 27 \mathrm{~A}$ & $0.5917(9)$ & $0.8484(4)$ & $0.2734(4)$ & $4.5(5)$ & $\mathrm{C} 27 \mathrm{C}$ & $0.055(1)$ & $0.3599(4)$ & $0.9606(4)$ & $6.0(6)$ \\
\hline $\mathrm{C} 28 \mathrm{~A}$ & $0.600(2)$ & $0.8927(7)$ & $0.2429(7)$ & $5(1)$ & $\mathrm{C} 28 \mathrm{C}$ & $0.064(1)$ & $0.4287(5)$ & $0.9974(7)$ & $10(1)$ \\
\hline $\mathrm{C} 28 \mathrm{~A}^{\prime}$ & $0.586(3)$ & $0.828(1)$ & $0.207(1)$ & $6.1(6)$ & $\mathrm{C} 29 \mathrm{C}$ & $0.098(1)$ & $0.4571(5)$ & $1.0652(5)$ & $7.4(7)$ \\
\hline $\mathrm{C} 29 \mathrm{~A}$ & $0.495(1)$ & $0.8794(4)$ & $0.1950(4)$ & $6.2(6)$ & $\mathrm{C} 30 \mathrm{C}$ & $0.597(1)$ & $0.3136(4)$ & $0.9400(4)$ & $6.0(6)$ \\
\hline C $30 A$ & $0.141(1)$ & $0.9409(4)$ & $0.4102(4)$ & $4.6(5)$ & $\mathrm{C} 31 \mathrm{C}$ & $0.650(1)$ & $0.3810(5)$ & $0.9721(5)$ & $7.7(8)$ \\
\hline C31A & $0.008(1)$ & $0.9455(4)$ & $0.3763(4)$ & $5.5(6)$ & $\mathrm{C} 32 \mathrm{C}$ & $0.642(1)$ & $0.4128(5)$ & $1.0442(4)$ & $6.0(6)$ \\
\hline $\mathrm{C} 32 \mathrm{~A}$ & $0.035(1)$ & $0.9701(4)$ & $0.3294(4)$ & $5.8(6)$ & FelD & $0.8146(1)$ & $0.34353(5)$ & $0.23519(5)$ & $3.02(6)$ \\
\hline FelB & $0.4583(1)$ & $0.15935(5)$ & $0.47787(5)$ & $3.23(6)$ & $\mathrm{Fe} 2 \mathrm{D}$ & $0.7521(2)$ & $0.12956(5)$ & $0.21734(5)$ & $3.88(7)$ \\
\hline $\mathrm{Fe} 2 \mathrm{~B}$ & $0.2241(1)$ & $0.36167(5)$ & $0.52100(5)$ & $3.31(7)$ & C1D & $0.8531(8)$ & $0.2665(3)$ & $0.2468(3)$ & $3.1(5)$ \\
\hline $\mathrm{C} 1 \mathrm{~B}$ & $0.4405(8)$ & $0.2507(3)$ & $0.5026(3)$ & $2.7(4)$ & C2D & $0.7463(9)$ & $0.3058(3)$ & $0.2896(3)$ & $3.3(5)$ \\
\hline $\mathrm{C} 2 \mathrm{~B}$ & $0.4134(8)$ & $0.2376(3)$ & $0.5534(3)$ & $3.1(4)$ & C3D & $0.8154(9)$ & $0.3643(4)$ & $0.3235(3)$ & $3.8(5)$ \\
\hline $\mathrm{C} 3 \mathrm{~B}$ & $0.5427(9)$ & $0.2103(3)$ & $0.5601(4)$ & $3.5(4)$ & C4D & $0.9590(9)$ & $0.3635(3)$ & $0.3005(4)$ & $3.6(5)$ \\
\hline $\mathrm{C} 4 \mathrm{~B}$ & $0.6432(8)$ & $0.2037(4)$ & $0.5123(4)$ & $3.8(5)$ & C5D & $0.9821(9)$ & $0.3016(3)$ & $0.2533(4)$ & $3.7(5)$ \\
\hline C5B & $0.5813(8)$ & $0.2310(3)$ & $0.4785(4)$ & $3.4(5)$ & C6D & $0.6266(9)$ & $0.3602(4)$ & $0.2076(4)$ & $4.1(5)$ \\
\hline $\mathrm{C} 6 \mathrm{~B}$ & $0.2926(8)$ & $0.1051(4)$ & $0.4750(4)$ & $4.1(5)$ & C7D & $0.7044(9)$ & $0.4162(4)$ & $0.2423(4)$ & $4.0(5)$ \\
\hline $\mathrm{C} 7 \mathrm{~B}$ & $0.4244(9)$ & $0.0801(4)$ & $0.4836(4)$ & $4.2(5)$ & C8D & $0.8427(9)$ & $0.4156(3)$ & $0.2152(4)$ & $3.7(5)$ \\
\hline $\mathrm{C} 8 \mathrm{~B}$ & $0.5208(9)$ & $0.0705(4)$ & $0.4336(4)$ & $4.5(5)$ & C9D & $0.8471(9)$ & $0.3582(4)$ & $0.1602(4)$ & $3.9(5)$ \\
\hline C9B & $0.447(1)$ & $0.0867(4)$ & $0.3932(4)$ & $4.8(5)$ & C10D & $0.7160(9)$ & $0.3152(3)$ & $0.1561(3)$ & $3.6(5)$ \\
\hline $\mathrm{ClOB}$ & $0.3093(9)$ & $0.1068(4)$ & $0.4181(4)$ & $4.4(5)$ & C11D & $0.8371(9)$ & $0.1996(3)$ & $0.2018(3)$ & $3.3(5)$ \\
\hline Cl1B & $0.3407(9)$ & $0.2814(3)$ & $0.4774(3)$ & $3.4(4)$ & C12D & $0.9399(9)$ & $0.1521(4)$ & $0.1896(4)$ & $3.8(5)$ \\
\hline $\mathrm{Cl} 2 \mathrm{~B}$ & $0.3835(9)$ & $0.3273(3)$ & $0.4585(3)$ & $3.6(5)$ & C13D & $0.8809(1)$ & $0.0964(4)$ & $0.1433(4)$ & $4.3(5)$ \\
\hline $\mathrm{Cl} 3 \mathrm{~B}$ & $0.2620(9)$ & $0.3413(4)$ & $0.4333(4)$ & $4.0(5)$ & $\mathrm{C} 14 \mathrm{D}$ & $0.7428(9)$ & $0.1070(3)$ & $0.1286(3)$ & $3.7(5)$ \\
\hline $\mathrm{Cl} 14 \mathrm{~B}$ & $0.1393(9)$ & $0.3081(4)$ & $0.4393(3)$ & $3.9(5)$ & C15D & $0.7164(9)$ & $0.1716(3)$ & $0.1637(3)$ & $3.3(5)$ \\
\hline C15B & $0.1886(9)$ & $0.2698(3)$ & $0.4656(4)$ & $3.6(5)$ & $\mathrm{C} 16 \mathrm{D}$ & $0.808(1)$ & $0.1133(4)$ & $0.2868(4)$ & $5.0(6)$ \\
\hline $\mathrm{C} 16 \mathrm{~B}$ & $0.3102(9)$ & $0.4416(4)$ & $0.5818(4)$ & $4.1(5)$ & C17D & $0.747(1)$ & $0.0586(4)$ & $0.2384(4)$ & $5.5(6)$ \\
\hline $\mathrm{C} 17 \mathrm{~B}$ & $0.187(1)$ & $0.4533(4)$ & $0.5547(4)$ & $4.7(6)$ & $\mathrm{C} 18 \mathrm{D}$ & $0.608(1)$ & $0.0713(4)$ & $0.2279(4)$ & $4.7(6)$ \\
\hline $\mathrm{C} 18 \mathrm{~B}$ & $0.0652(9)$ & $0.4212(4)$ & $0.5630(4)$ & $4.1(5)$ & C19D & $0.580(1)$ & $0.1341(4)$ & $0.2723(4)$ & $5.2(6)$ \\
\hline C19B & $0.1070(9)$ & $0.3943(4)$ & $0.6008(4)$ & $4.1(5)$ & C20D & $0.702(1)$ & $0.1596(4)$ & $0.3083(4)$ & $4.8(6)$ \\
\hline $\mathrm{C} 20 \mathrm{~B}$ & $0.2564(9)$ & $0.4063(4)$ & $0.6119(3)$ & $4.1(5)$ & C21D & $0.5872(9)$ & $0.2935(4)$ & $0.2948(3)$ & $3.8(5)$ \\
\hline $\mathrm{C} 21 \mathrm{~B}$ & $0.2746(9)$ & $0.2419(4)$ & $0.5893(4)$ & $3.9(5)$ & C22D & $0.485(1)$ & $0.3400(4)$ & $0.2893(4)$ & $5.1(6)$ \\
\hline $\mathrm{C} 22 \mathrm{~B}$ & $0.221(1)$ & $0.1808(4)$ & $0.5869(4)$ & $5.2(6)$ & C23D & $0.4862(9)$ & $0.3394(4)$ & $0.2264(4)$ & $4.8(6)$ \\
\hline $\mathrm{C} 23 \mathrm{~B}$ & $0.1737(9)$ & $0.1310(4)$ & $0.5244(5)$ & $5.5(6)$ & C24D & $1.060(1)$ & $0.4187(4)$ & $0.3170(4)$ & $4.9(5)$ \\
\hline $\mathrm{C} 24 \mathrm{~B}$ & $0.781(1)$ & $0.1691(4)$ & $0.4959(4)$ & $5.7(6)$ & C25D & $1.089(1)$ & $0.4372(5)$ & $0.2660(5)$ & $6.6(7)$ \\
\hline $\mathrm{C} 25 \mathrm{~B}$ & $0.781(1)$ & $0.1081(5)$ & $0.4457(6)$ & $8.2(8)$ & $\mathrm{C} 26 \mathrm{D}$ & $0.963(1)$ & $0.4616(4)$ & $0.2438(4)$ & $5.0(6)$ \\
\hline $\mathrm{C} 26 \mathrm{~B}$ & $0.678(1)$ & $0.0563(4)$ & $0.4299(5)$ & $6.5(6)$ & C27D & $1.074(1)$ & $0.1563(4)$ & $0.2251(4)$ & $5.5(6)$ \\
\hline $\mathrm{C} 27 \mathrm{~B}$ & $0.528(1)$ & $0.3617(4)$ & $0.4715(4)$ & $4.9(6)$ & $\mathrm{C} 28 \mathrm{D}$ & $1.072(1)$ & $0.1196(7)$ & $0.2606(7)$ & $11(1)$ \\
\hline $\mathrm{C} 28 \mathrm{~B}$ & $0.521(1)$ & $0.4316(4)$ & $0.5093(5)$ & $6.6(7)$ & C29D & $0.959(1)$ & $0.1224(4)$ & $0.3052(4)$ & $6.8(7)$ \\
\hline $\mathrm{C} 29 \mathrm{~B}$ & $0.465(1)$ & $0.4580(4)$ & $0.5751(4)$ & $5.2(6)$ & C30D & $0.633(1)$ & $0.0585(4)$ & $0.0915(4)$ & $5.2(6)$ \\
\hline $\mathrm{C} 30 \mathrm{~B}$ & $-0.017(1)$ & $0.3177(4)$ & $0.4294(4)$ & $5.1(6)$ & C31D & $0.497(1)$ & $0.0534(4)$ & $0.1260(4)$ & $5.9(6)$ \\
\hline $\mathrm{C} 31 \mathrm{~B}$ & $-0.068(1)$ & $0.3851(5)$ & $0.4640(4)$ & $5.8(6)$ & C32D & $0.517(1)$ & $0.0308(4)$ & $0.1741(5)$ & $6.2(7)$ \\
\hline
\end{tabular}

anisotropically. During the final cycles of refinement fixed hydrogen contributions with $\mathrm{C}-\mathrm{H}$ bond lengths held at $1.08 \AA$ were applied. The final positional parameters for all atoms can be found in Table 2, and the selected bond distances and angles are given in Table 3.

Structure Determination of $17 \mathrm{~b}$. A orange crystal $(0.43 \times$ $0.18 \times 0.19 \mathrm{~mm}$ ) was obtained by following the same procedure as described for $17 \mathrm{a}$. Data were collected to a $2 \theta$ value of $44.8^{\circ}$. The unit cell dimensions were obtained from 20 reflections with $18.93^{\circ}<2 \theta<29.55^{\circ}$. Of the 9860 unique reflections, there were 5334 reflections with $F_{0}>2.0 \sigma\left(F_{0}{ }^{2}\right)$. These data were used in the final refinement of structural parameters. The structure was solved by the heavy-atom method to locate the Fe positions and successive Fourier maps to reveal the whole molecule and refined 
Table 5. Selected Bond Distances $(\boldsymbol{A})$ and Bond Angles (deg) for 17b

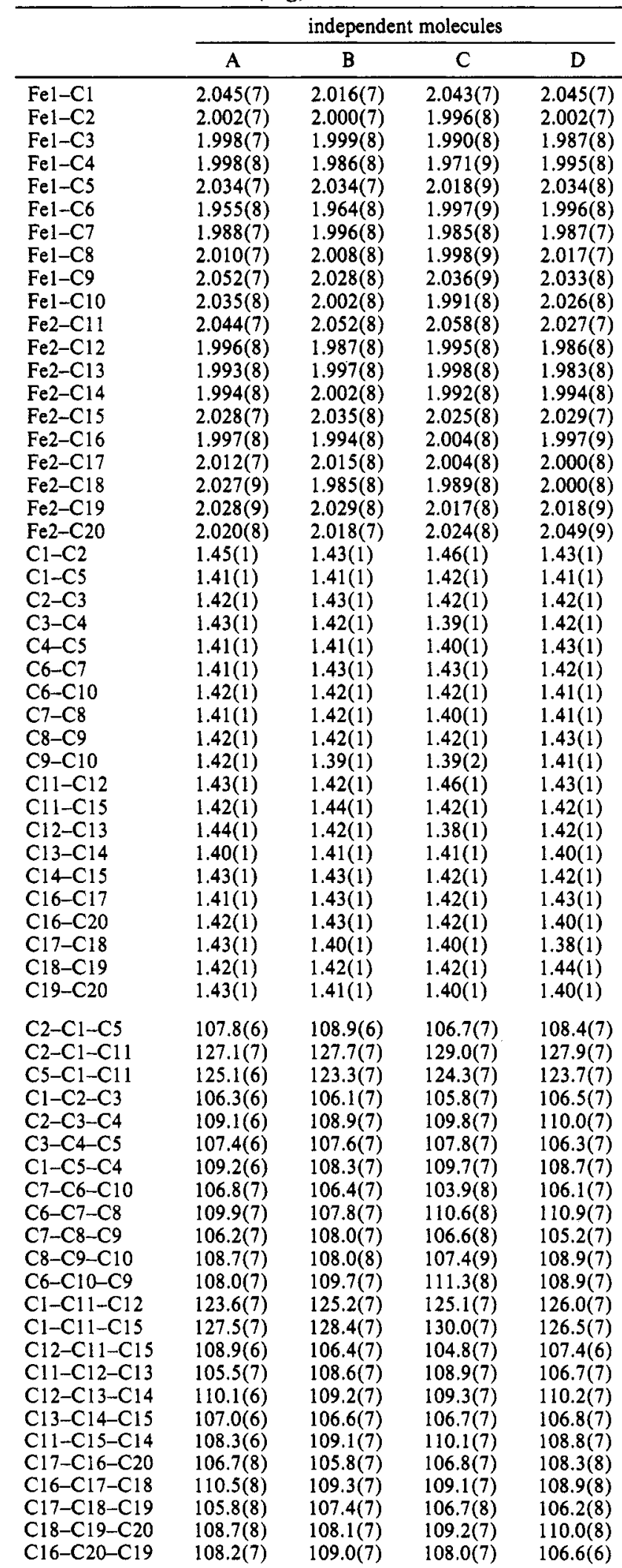

with a weighted least-squares routine. Atomic coordinates and selected bond distances and angles are given in Tables 4 and 5, respectively.

Structure Determination of 18a. A black crystal $(0.20 \times$ $0.13 \times 0.25 \mathrm{~mm}$ ) was obtained when a layer of hexane was allowed to slowly diffuse into a $\mathrm{CH}_{2} \mathrm{Cl}_{2}$ solution of $18 \mathrm{a}$. Data were collected to a $2 \theta$ value of $44.9^{\circ}$. The cell dimensions were obtained from 25 reflections with $2 \theta$ in the range $14.65-34.40^{\circ}$. Of the 3866
Table 6. Atom Coordinates and Thermal Parameters $\left(\AA^{2}\right)$ for $18 a$

\begin{tabular}{lllll}
\hline & \multicolumn{1}{c}{$x$} & \multicolumn{1}{c}{$y$} & \multicolumn{1}{c}{$z$} & \multicolumn{1}{c}{$B_{\text {iso }}{ }^{2}$} \\
\hline I1 & $0.36281(8)$ & $0.77830(7)$ & $0.16700(4)$ & $4.55(4)$ \\
I2 & $0.3787(1)$ & $0.57391(8)$ & $0.20883(5)$ & $5.99(5)$ \\
I3 & $0.3381(1)$ & $0.98030(8)$ & $0.11914(6)$ & $6.63(6)$ \\
Fe1 & $0.9400(2)$ & $0.8024(1)$ & $0.19862(8)$ & $3.00(7)$ \\
Fe2 & $1.2914(2)$ & $0.8331(1)$ & $0.40646(8)$ & $2.84(7)$ \\
C1 & $1.0415(9)$ & $0.8496(7)$ & $0.2937(5)$ & $2.7(5)$ \\
C2 & $0.912(1)$ & $0.8679(8)$ & $0.2847(6)$ & $3.8(6)$ \\
C3 & $0.876(1)$ & $0.9329(8)$ & $0.2223(6)$ & $3.6(6)$ \\
C4 & $0.979(1)$ & $0.9452(7)$ & $0.1929(6)$ & $3.8(6)$ \\
C5 & $1.080(1)$ & $0.8950(8)$ & $0.2370(6)$ & $3.3(5)$ \\
C6 & $0.817(1)$ & $0.6924(9)$ & $0.1846(6)$ & $4.1(6)$ \\
C7 & $0.789(1)$ & $0.761(1)$ & $0.1276(6)$ & $4.5(6)$ \\
C8 & $0.887(1)$ & $0.769(1)$ & $0.0968(6)$ & $4.9(7)$ \\
C9 & $0.979(1)$ & $0.7015(9)$ & $0.1319(6)$ & $4.4(6)$ \\
C10 & $0.937(1)$ & $0.6563(8)$ & $0.1837(6)$ & $4.3(6)$ \\
C11 & $1.1173(9)$ & $0.7906(7)$ & $0.3484(5)$ & $2.4(5)$ \\
C12 & $1.2113(9)$ & $0.7226(8)$ & $0.3394(5)$ & $2.8(5)$ \\
C13 & $1.2725(9)$ & $0.6861(7)$ & $0.4065(5)$ & $2.7(5)$ \\
C14 & $1.218(1)$ & $0.7291(7)$ & $0.4561(5)$ & $2.7(5)$ \\
C15 & $1.1249(9)$ & $0.7975(7)$ & $0.4223(5)$ & $2.8(5)$ \\
C16 & $1.476(1)$ & $0.8470(9)$ & $0.4449(6)$ & $3.9(6)$ \\
C17 & $1.416(1)$ & $0.8831(8)$ & $0.4955(6)$ & $3.5(6)$ \\
C18 & $1.334(1)$ & $0.9606(8)$ & $0.4652(6)$ & $3.7(6)$ \\
C19 & $1.350(1)$ & $0.9742(9)$ & $0.3967(7)$ & $4.9(7)$ \\
C20 & $1.433(1)$ & $0.970(1)$ & $0.3833(6)$ & $4.6(6)$ \\
C21 & $0.827(1)$ & $0.8228(9)$ & $0.3216(6)$ & $3.9(6)$ \\
C22 & $0.718(1)$ & $0.769(1)$ & $0.2741(7)$ & $6.0(8)$ \\
C23 & $0.747(1)$ & $0.676(1)$ & $0.2375(7)$ & $5.3(7)$ \\
C24 & $0.976(1)$ & $0.9928(9)$ & $0.1240(7)$ & $5.1(7)$ \\
C25 & $0.892(2)$ & $0.948(1)$ & $0.0632(9)$ & $9(1)$ \\
C26 & $0.904(2)$ & $0.843(1)$ & $0.0449(6)$ & $6.7(9)$ \\
C27 & $1.387(1)$ & $0.6222(8)$ & $0.4230(6)$ & $4.1(6)$ \\
C28 & $1.496(1)$ & $0.6645(9)$ & $0.4773(6)$ & $4.4(6)$ \\
C29 & $1.552(1)$ & $0.757(1)$ & $0.4514(6)$ & $4.5(7)$ \\
C30 & $1.065(1)$ & $0.8726(9)$ & $0.4577(6)$ & $3.8(6)$ \\
C31 & $1.154(1)$ & $0.9289(9)$ & $0.5177(6)$ & $3.9(6)$ \\
C32 & $1.237(1)$ & $1.0028(9)$ & $0.4948(7)$ & $4.3(6)$ \\
& & &
\end{tabular}

${ }^{a} B_{\text {iso }}$ is the mean of the principal axes of the thermal ellipsoid.

unique reflections, there were 2296 reflections with $F_{0}^{2}>2.0 \sigma$ $\left(F_{0}^{2}\right)$. These data were used in the final refinement of the structural parameters.

Structure refinement was carried out in the same manner as described for 17a. The greatest residual electron density upon completion of refinement was in the vicinity of the iodide atoms. Selected bond distances and angles and atomic coordinates are given in Tables 3 and 6 , respectively.

Structure Determination of $18 b$. A black crystal $(0.38 \times$ $0.09 \times 0.19 \mathrm{~mm}$ ) was obtained when a layer of ether was allowed to slowly diffuse into a $\mathrm{CH}_{2} \mathrm{Cl}_{2}$ solution of $18 \mathbf{b}$. Data were collected to a $2 \theta$ value of $49.8^{\circ}$. The cell dimensions were obtained from 25 reflections with $14.72<2 \theta<31.02$. Of the 5197 unique reflections, there were 3727 reflections with $F_{0}{ }^{2}>2.5 \sigma\left(F_{0}{ }^{2}\right)$. These data were used in the final refinement of the structural parameters.

Structure refinement was carried out in the same manner as described for 18a. The greatest residual electron density upon completion of refinement was in the vicinity of the iodide atoms. Selected bond distances and angles and atomic coordinates are given in Tables 3 and 7, respectively.

\section{Results and Discussion}

Although the physical properties of $17 \mathrm{a}$ and $17 \mathrm{~b}$ are different, it is difficult to assign their structures with certainty solely with NMR spectroscopy. X-ray crystallographic studies of these complexes were undertaken to help elucidate the structures and geometric influences on the rate of intramolecular electron transfer in mixedvalence biferrocenium cations. Before the new physical data are described, a summary of single-crystal X-ray structural results obtained for $17 \mathbf{a}, \mathbf{b}$ and $18 \mathbf{a}, \mathbf{b}$ will be presented. 
Table 7. Atom Coordinates and Thermal Parameters $\left(\AA^{2}\right)$ for $18 b$

\begin{tabular}{|c|c|c|c|c|}
\hline & $x$ & $y$ & $z$ & $B_{\text {iso }}{ }^{a}$ \\
\hline 11 & $0.77102(3)$ & $0.22273(4)$ & $0.07191(2)$ & $3.91(2)$ \\
\hline 12 & $0.96402(4)$ & $0.07792(4)$ & $0.10834(2)$ & $4.53(2)$ \\
\hline 13 & $0.57734(5)$ & $0.35503(5)$ & $0.02767(3)$ & $6.86(3)$ \\
\hline Fel & $0.76711(6)$ & $0.73016(6)$ & $0.14809(3)$ & $2.19(3)$ \\
\hline $\mathrm{Fe} 2$ & $0.36933(6)$ & $0.86697(6)$ & $0.20829(3)$ & $2.34(3)$ \\
\hline $\mathrm{Cl}$ & $0.6015(4)$ & $0.8074(4)$ & $0.1427(2)$ & $2.3(2)$ \\
\hline $\mathrm{C} 2$ & $0.5996(4)$ & $0.6895(4)$ & $0.1309(2)$ & $2.3(2)$ \\
\hline C3 & $0.6690(4)$ & $0.6696(4)$ & $0.0769(2)$ & $2.6(2)$ \\
\hline $\mathrm{C} 4$ & $0.7236(4)$ & $0.7693(5)$ & $0.0577(2)$ & $2.8(2)$ \\
\hline C5 & $0.6806(4)$ & $0.8541(4)$ & $0.0981(2)$ & $2.6(2)$ \\
\hline C6 & $0.8140(4)$ & $0.6115(4)$ & $0.2114(2)$ & $2.8(2)$ \\
\hline C7 & $0.8704(4)$ & $0.5966(4)$ & $0.1525(2)$ & $2.8(2)$ \\
\hline $\mathrm{C} 8$ & $0.9373(4)$ & $0.6910(5)$ & $0.1370(2)$ & $2.9(2)$ \\
\hline C9 & $0.9278(4)$ & $0.7643(5)$ & $0.1903(2)$ & $3.2(2)$ \\
\hline $\mathrm{ClO}$ & $0.8540(5)$ & $0.7153(5)$ & $0.2345(2)$ & $3.1(2)$ \\
\hline $\mathrm{C} 11$ & $0.5419(4)$ & $0.8690(4)$ & $0.1917(2)$ & $2.5(2)$ \\
\hline $\mathrm{C} 12$ & $0.5182(4)$ & $0.8348(4)$ & $0.2554(2)$ & $2.6(2)$ \\
\hline $\mathrm{Cl} 3$ & $0.4534(4)$ & $0.9188(5)$ & $0.2860(2)$ & $2.9(2)$ \\
\hline $\mathrm{Cl} 4$ & $0.4395(4)$ & $1.0071(4)$ & $0.2414(3)$ & $2.7(2)$ \\
\hline $\mathrm{C} 15$ & $0.4900(4)$ & $0.9773(4)$ & $0.1825(2)$ & $2.5(2)$ \\
\hline $\mathrm{Cl} 6$ & $0.2140(4)$ & $0.8276(5)$ & $0.2439(3)$ & $3.6(3)$ \\
\hline $\mathrm{C} 17$ & $0.2045(4)$ & $0.9182(5)$ & $0.2008(3)$ & $3.3(3)$ \\
\hline $\mathrm{C} 18$ & $0.2493(4)$ & $0.8892(5)$ & $0.1406(3)$ & $3.4(3)$ \\
\hline $\mathrm{C} 19$ & $0.2797(5)$ & $0.7753(5)$ & $0.1442(3)$ & $3.9(3)$ \\
\hline $\mathrm{C} 20$ & $0.2582(5)$ & $0.7379(5)$ & $0.2068(3)$ & $3.8(3)$ \\
\hline C21 & $0.5435(4)$ & $0.5990(4)$ & $0.1696(3)$ & $3.0(2)$ \\
\hline $\mathrm{C} 22$ & $0.6256(5)$ & $0.5072(4)$ & $0.1919(3)$ & $3.5(2)$ \\
\hline $\mathrm{C} 23$ & $0.7220(5)$ & $0.5401(5)$ & $0.2388(3)$ & $3.5(2)$ \\
\hline C24 & $0.8179(5)$ & $0.7805(5)$ & $0.0094(2)$ & $3.5(2)$ \\
\hline $\mathrm{C} 25$ & $0.9171(5)$ & $0.6975(5)$ & $0.0171(3)$ & $3.6(2)$ \\
\hline $\mathrm{C} 26$ & $0.9948(5)$ & $0.7149(5)$ & $0.0752(3)$ & $3.6(3)$ \\
\hline C27 & $0.3955(5)$ & $0.9088(5)$ & $0.3492(3)$ & $3.8(3)$ \\
\hline C28 & $0.2653(6)$ & $0.9167(6)$ & $0.3497(3)$ & $5.1(3)$ \\
\hline C29 & $0.1983(6)$ & $0.8291(6)$ & $0.3141(3)$ & $5.0(3)$ \\
\hline C30 & $0.4737(5)$ & $1.0392(5)$ & $0.1218(3)$ & $3.6(2)$ \\
\hline C31 & $0.3969(6)$ & $0.9858(7)$ & $0.0725(3)$ & $5.9(4)$ \\
\hline C 32 & $0.2729(5)$ & $0.9670(6)$ & $0.0875(3)$ & $4.5(3)$ \\
\hline
\end{tabular}

Molecular Structure of $17 \mathrm{a}$. The results of our crystallographic study at room temperature show that 17a crystallizes in the triclinic space group $P \overline{1}$. As shown in Figure 1, there are two crystallographically independent molecules in the unit cell. Collected in Table 3 are selected bond distances and angles. The two independent molecules have very similar geometrical arrangements. However, they do not adopt the usual trans conformation observed in the series of dialkylbiferrocenium cations 1-16. 7,15 The two $\mathrm{Cp}$ rings in the fulvalenide bridge are not coplanar, and the dihedral angles between the two $\mathrm{Cp}$ rings of the fulvalenide ligand in the two independent molecules labeled as $\mathrm{FeA}$ and $\mathrm{FeB}$ are 58.7(2) and 53.6$(2)^{\circ}$, respectively. Inspection of the iron to $\mathrm{Cp}$ ligand bond lengths $(\mathrm{Fe}-\mathrm{Cp})$ shows that the average distance $(1.603(3)$ $\AA$ ) is significantly less than the values of 1.65 and $1.70 \AA$ found for ferrocene ${ }^{30}$ and ferrocenium, ${ }^{31}$ respectively. This is mainly due to the compression of the interannular trimethylene bridge on the $\mathrm{Fe}$ atom. The dihedral angles between the two least-squares planes of the $\mathrm{Cp}$ rings respectively associated with $\mathrm{Fe} 1 \mathrm{~A}$ and $\mathrm{Fe} 2 \mathrm{~A}$ are 9.9(2) and $10.9(2)^{\circ}$, while the two Cp rings in each ferrocenyl moiety are nearly eclipsed with average staggering angles of $0.3(3)$ and $0.5(3)^{\circ}$. The two Cp rings associated with $\mathrm{Fe} 1 \mathrm{~B}$ and $\mathrm{Fe} 2 \mathrm{~B}$ are also nearly eclipsed with average

(30) Seiler, P.; Dunitz, J. D. Acta Crystallogr., Sect. B 1979, 35, 1068. (31) Mammano, N. J.; Zalkin, A.; Landers, A.; Rheingold, A. L. Inorg. Chem. 1977, 16, 297.

(32) Dong, T.-Y.; Hwang, M. Y.; Wen, Y. S. J. Organomet. Chem. $1990,391,377$.
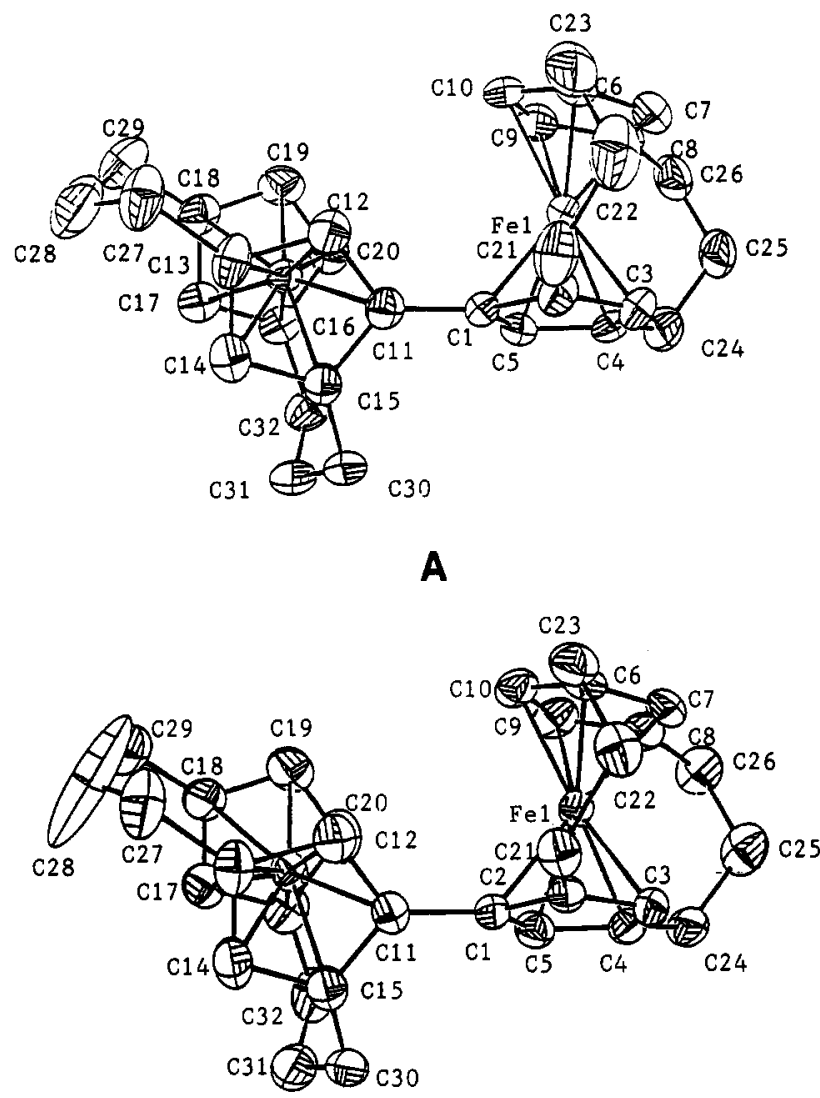

B

Figure 1. ORTEP drawings for the two independent molecules (A and B) of $17 \mathrm{a}$ with $30 \%$ thermal ellipsoids.

staggering angles of $0.5(3)$ and $0.4(3)^{\circ}$. Furthermore, the two planes of the $\mathrm{Cp}$ rings bonded to $\mathrm{Fe} 1 \mathrm{~B}$ and $\mathrm{Fe} 2 \mathrm{~B}$ form dihedral angles of $11.4(2)$ and $9.0(2)^{\circ}$ with the fulvalenide plane, respectively. The average $\mathrm{Fe}-\mathrm{C}$ bond distance of $2.011(5) \AA$ is less than the values of 2.045 and $2.075 \AA$ found for the ferrocene ${ }^{30}$ and ferrocenium ions, ${ }^{31}$ respectively. The average $\mathrm{C}-\mathrm{C}$ bond length $(1.426(8) \AA)$ in the $\mathrm{Cp}$ rings agrees well with that in ferrocene $(1.42 \AA){ }^{30}$

Molecular Structure of $17 \mathrm{~b}$. Compound $17 \mathrm{~b}$ crystallizes at room temperature in the triclinic space group $P \overline{1}$. There are four crystallographically independent molecules in the unit cell, and the molecular structures are shown in Figure 2. There is a very similar geometrical arrangement in the four independent molecules. Atomic coordinates and important bond distances and angles are given in Tables 4 and 5 , respectively. As found in compound $17 \mathrm{a}$, compound $17 \mathrm{~b}$ also does not adopt the usual trans conformation seen in the series of disubstituted biferrocenium cations 1-16.7,15 The dihedral angles between the two least-squares $\mathrm{Cp}$ planes of fulvalenide ligands in four independent molecules respectively labeled as A-D in Figure 2 are 53.2(4), 49.1(4), 46.9(4), and 51.4(4) ${ }^{\circ}$. The average $\mathrm{Fe}-\mathrm{Cp}$ and $\mathrm{Fe}-\mathrm{C}$ bond lengths are 1.606(4) and $2.009(8) \AA$, respectively. Thus, the average bond distance between the iron atom and the five carbons for a given ring and the average $\mathrm{Fe}-\mathrm{Cp}$ distance for a ferrocenyl moiety are very similar in both compounds $17 \mathrm{a}$ and $17 \mathbf{b}$. The two Cp rings in each ferrocenyl moiety are nearly eclipsed with an average staggering angle of $1.4(5)^{\circ}$, while the average dihedral angle between these two $\mathrm{Cp}$ rings is $9.9(4)^{\circ}$. The 


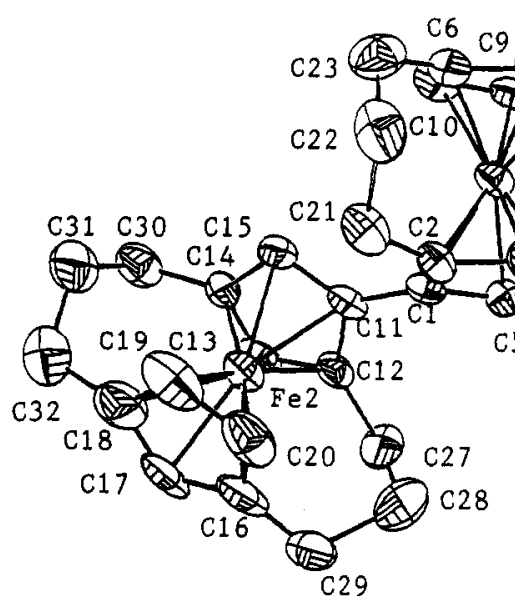

A

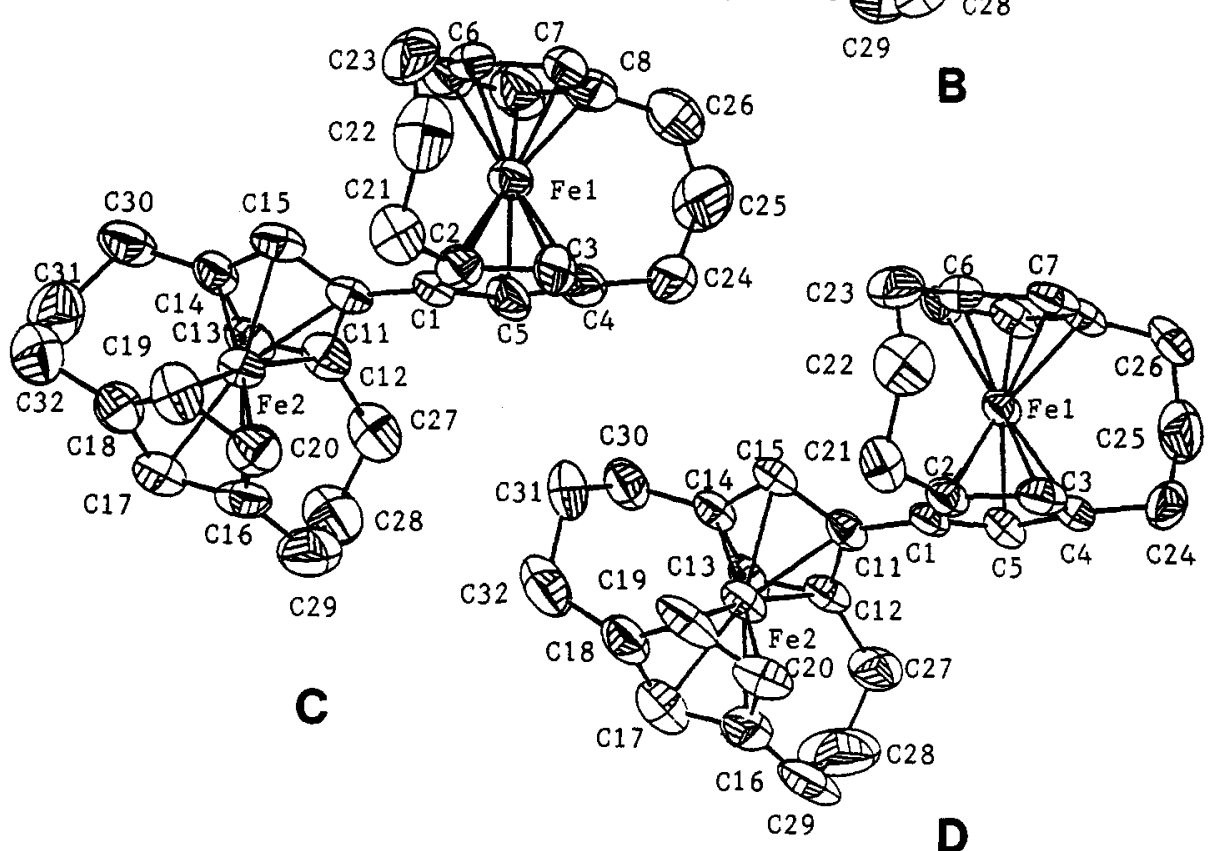

Figure 2. ORTEP drawings for the four independent molecules (A-D) of $\mathbf{1 7 b}$ with $30 \%$ thermal ellipsoids.

average $\mathrm{C}-\mathrm{C}$ bond length (1.42(1) $\AA$ ) in the $\mathrm{Cp}$ rings also agrees well with that in ferrocene $(1.42 \AA){ }^{30}$

Molecular Structure of 18a. A drawing of the mixedvalence compound 18a is shown in Figure 3. Important bond distances and angles are collected in Table 3 . The two metallocene moieties in the cation are not equivalent. In comparison with ferrocenes ${ }^{30}$ and $17 \mathrm{a}$, the average $\mathrm{Fe} 1-$ Cp distance (1.608(6) $\AA$ ) and the average Fe1-C interatomic distance $(2.02(1) \AA)$ indicate that the $\mathrm{Fe} 1(\mathrm{Cp})_{2}$ metallocene unit is in the $\mathrm{Fe}$ (II) oxidation state. However, the average $\mathrm{Fe} 2-\mathrm{Cp}$ distance $(1.658(6) \AA)$ and the average $\mathrm{Fe} 2-\mathrm{C}$ distance $(2.06(1) \AA)$, which are larger than the corresponding values in $17 \mathrm{a}$, indicate that the $\mathrm{Fe} 2(\mathrm{Cp})_{2}$ unit is the Fe(III) metallocene moiety. Such an increase in $\mathrm{Fe}-\mathrm{Cp}$ and $\mathrm{Fe}-\mathrm{C}$ distances has been observed when ferrocenes are oxidized to the corresponding ferrocenium cations. ${ }^{18,31}$ There is no significant difference for $\mathrm{C}-\mathrm{C}$ bond distances in the $\mathrm{Cp}$ rings between two nonequivalent $\mathrm{Fe}$ (II) and $\mathrm{Fe}$ (III) metallocene moieties. The observation of two nonequivalent $\mathrm{Fe}$ (II) and $\mathrm{Fe}$ (III) moieties is consistent with our Mössbauer studies.

The conformational relationship between two iron metallocene moieties plays an important role in determin- ing the magnitude of electron-transfer rate. As can be seen in Figure 3 , the two metallocene units are twisted relative to each other. The dihedral angle between the two $\mathrm{Cp}$ rings in the fulvalenide ligand is $42.0(5)^{\circ}$. The two $\mathrm{Cp}$ rings associated with $\mathrm{Fel}$ are not quite parallel, with a dihedral angle of $11.2(5)^{\circ}$. The dihedral angle between the two $\mathrm{Cp}$ rings associated with $\mathrm{Fe} 2$ is $14.6(5)^{\circ}$. Furthermore, the two $\mathrm{Cp}$ rings associated with $\mathrm{Fel}$ and Fe2 are nearly eclipsed with average angles of $0.8(7)$ and $2.1(7)^{\circ}$, respectively.

The triiodide anion in $18 \mathrm{a}$ is essentially linear, and the I2-I1-I3 angle is $177.26(4)^{\circ}$. The triiodide anion also shows an essentially symmetric structure. The distance from I1 to $\mathrm{I} 2$ is $2.905(2) \AA$, while the distance from I1 to I3 is 2.908(2) $\AA$.

Molecular Structure of $18 \mathrm{~b}$. The results of our crystallographic study at room temperature show that $\mathbf{1 8 b}$ crystallizes in the monoclinic space group $P 2_{1} / n$, and the molecular structure is shown in Figure 4. Collected in Table 3 are selected bond distances and angles. The two metallocene moieties in the cation are not equivalent. In comparison with $17 \mathrm{~b}$, the average $\mathrm{Fe} 1-\mathrm{Cp}$ distance (1.662(2) $\AA$ ) and the average Fe1-C distance $(2.059(5) \AA)$ indicate 


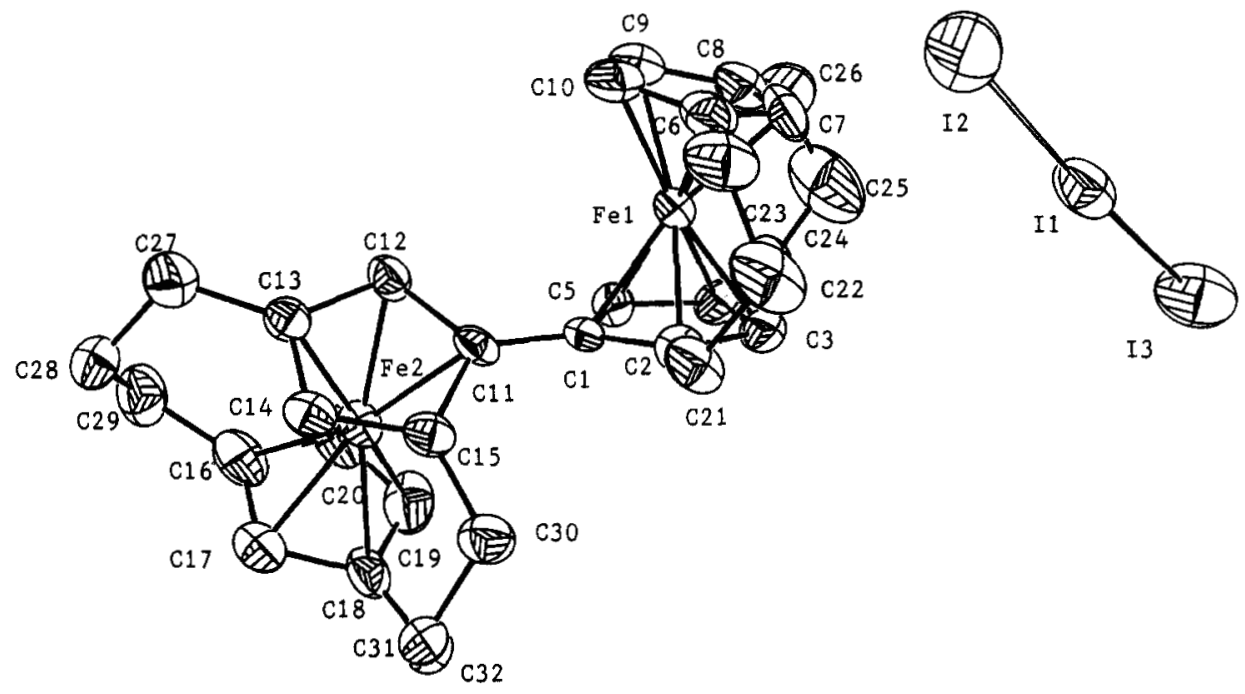

Figure 3. ORTEP drawing for 18 a with $30 \%$ thermal ellipsoids.

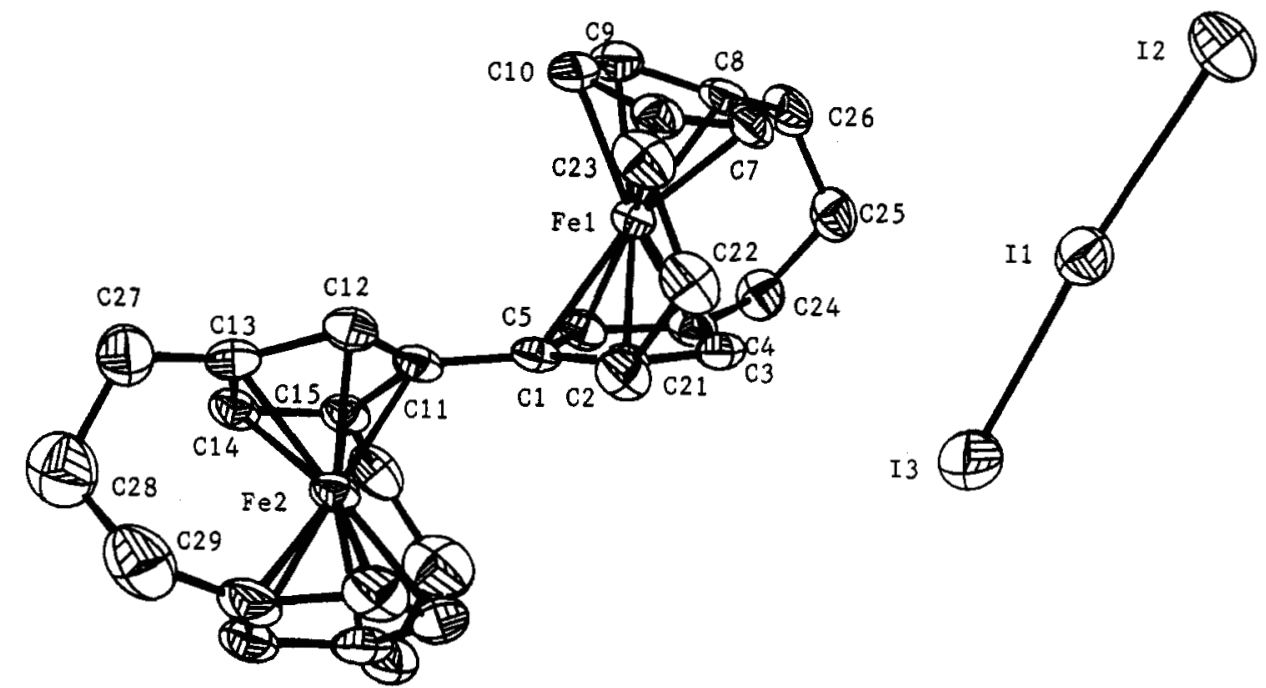

Figure 4. ORTEP drawing for $18 \mathrm{~b}$ with $30 \%$ thermal ellipsoids.

that this metallocene is in the $\mathrm{Fe}$ (III) oxidation state. The average $\mathrm{Fe} 2-\mathrm{Cp}$ distance $(1.608(2) \AA)$ and the average $\mathrm{Fe} 2-\mathrm{C}$ distance $(2.016(5) \AA)$ indicate that this metallocene is in the $\mathrm{Fe}$ (II) oxidation state. The observation of two nonequivalent $\mathrm{Fe}$ (II) and $\mathrm{Fe}$ (III) moieties is also consistent with our Mössbauer studies.

Similar to the geometrical arrangement for 18a, in $18 b$ the two metallocene units are also twisted relative to each other. The dihedral angle between the two Cp rings in the fulvalenide ligand is $38.80(22)^{\circ}$. The two $\mathrm{Cp}$ rings respectively associated with $\mathrm{Fe} 1$ and $\mathrm{Fe} 2$ are not quite parallel, with dihedral angles of $19.25(23)$ and $9.74(24)^{\circ}$. Furthermore, the two $\mathrm{Cp}$ rings associated with $\mathrm{Fel}$ and $\mathrm{Fe} 2$ are nearly eclipsed with average staggering angles of 0.4 and $1.6(4)^{\circ}$, respectively.

The triiodide anion in $18 \mathrm{~b}$ is slightly asymmetric (I1-I2 $=2.9393(6) \AA$ and $\mathrm{I} 1-\mathrm{I} 3=2.9058(7) \AA)$. The angle I2$\mathrm{I} 1-\mathrm{I} 3$ is $175.65(2)^{\circ}$.

Electrochemical Results. Electrochemical data for compounds $17 \mathrm{a}, \mathbf{b}$, as well as those for some other relevant compounds, are shown in Table 8. The neutral compounds $17 \mathbf{a}, \mathbf{b}$ undergo two successive reversible one-electron oxidations to yield the mono- and then the dication. Electrochemical reversibility was demonstrated by the peak-to-peak separation between the resolved reduction and oxidation wave maxima.
Table 8. Electrochemical Data for Various Biferrocenes

\begin{tabular}{lcccc}
\hline \multicolumn{1}{c}{ compd } & $E_{1 / 2}(\mathrm{~V})^{a}$ & $\Delta E_{1 / 2}(\mathrm{~V})^{b}$ & $\Delta(\mathrm{mV})^{c}$ & $10^{-5} K_{\text {com }}$ \\
\hline ferrocene & 0.37 & & 70 & \\
biferrocene & 0.28 & 0.31 & 70 & 1.80 \\
& 0.59 & & 75 & \\
$17 \mathrm{a}$ & 0.244 & 0.333 & 66 & 4.41 \\
& 0.577 & & 65 & \\
$17 \mathbf{b}$ & 0.246 & 0.326 & 65 & 3.35 \\
& 0.572 & & 74 &
\end{tabular}

a All half-wave potentials are referred to the $\mathrm{Ag}^{+} / \mathrm{Ag}$ electrode. ${ }^{b}$ Peak separation between two waves. ${ }^{c}$ Peak-to-peak separation between the resolved reduction and oxidation wave maxima.

In the case of biferrocenes, there are two possible types of interactions between the two ferrocene moieties: interaction propagated through the fulvalenide and through space (electric field effect). ${ }^{22}$ It is reasonable to believe that the interaction through the fulvalenide between two $\mathrm{Fe}$ sites is strongly favored. Furthermore, it has been shown that the magnitude of the peak-to-peak separation $\left(\Delta E_{1 / 2}\right)$ gives an indication of the interaction between the two Fe sites. ${ }^{33}$ A comparison of the magnitude of $\Delta E_{1 / 2}$ for $17 \mathrm{a}(0.333 \mathrm{~V})$ with that for $17 \mathrm{~b}(0.326 \mathrm{~V})$ indicates that the magnitude of interaction between the two $\mathrm{Fe}$ sites in

(33) Morrison, W. H., Jr.; Krogsrud, S.; Hendrickson, D. N. Inorg. Chem. 1973, 12, 1998 . 
$17 \mathrm{a}$ is similar to that in $\mathbf{1 7 b}$. However, a comparison of $\Delta E_{1 / 2}$ values for $17 \mathrm{a}, \mathrm{b}$ with that for biferrocene indicates that the interaction between the two Fe sites in $17 \mathbf{a}, \mathbf{b}$ is larger than that in biferrocene. As reported before, ${ }^{22,33}$ the conformational relationship between the two $\mathrm{Cp}$ rings in the fulvalenide bridge is critical to the magnitude of $\mathrm{Fe}-\mathrm{Fe}$ interactions in biferrocene compounds. In contrast to $17 \mathrm{a}, \mathrm{b}$, the two $\mathrm{Cp}$ rings of the fulvalenide bridge in biferrocene are crystallographically coplanar. ${ }^{34}$ Hence, it appears that in the solution state the two $\mathrm{Cp}$ rings of the fulvalenide bridge in $\mathbf{1 7 a}, \mathbf{b}$ have to be coplanar in order to have a larger $\mathrm{Fe}-\mathrm{Fe}$ interaction. This is different from the solid-state structures of $17 \mathbf{a}, \mathbf{b}$.

Coulometry experiments show that each of the two waves is a one-electron-transfer process. In eq 1 the abbreviation

$$
[2,2]+[3,3] \stackrel{K}{\rightleftharpoons} 2[2,3]
$$

$[3,3]$ denotes the dioxidized salt, $[2,3]$ the monooxidized salt, and $[2,2]$ the neutral compound. Comproportionation equilibrium constants $K$ for $17 \mathbf{a}, \mathbf{b}$ are $4.41 \times 10^{5}$ and 3.35 $\times 10^{5}$, respectively. Quantitative calculations based on the concentration of $[2,3]$ have been corrected for this equilibrium.

Electron Transfer in the Solution State. A description of the width of the intervalence transition band (IT band), the extent of electron delocalization, and the electron-transfer properties of mixed-valence dimers has been given by Hush. ${ }^{35}$ Hush has derived an expression for the bandwidth (in $\mathrm{cm}^{-1}$ ) at half-maximum of the IT band of a localized homonuclear mixed-valence dimer at $300 \mathrm{~K}$ as shown in eq 2 , where $\nu_{\max }$ is the frequency in $\mathrm{cm}^{-1}$

$$
\begin{gathered}
\Delta \nu_{1 / 2}=2310 \nu_{\max } \\
\Delta E^{*}=1 / 4^{\nu_{\max }} \\
\alpha^{2}=\left(4.24 \times 10^{-4}\right) \epsilon_{\max }\left(\Delta \nu_{1 / 2}\right) / \nu_{\max } d^{2} \\
H_{\mathrm{ab}}=\nu_{\max } \alpha \\
K_{\mathrm{et}}=\nu_{\mathrm{et}} \exp \left(-\nu_{\max } / 4 K_{\mathrm{B}} T\right) \\
\nu_{\mathrm{et}}=(2 \pi / \hbar) H_{\mathrm{ab}}{ }^{2}\left(\pi / K_{\mathrm{B}} T \nu_{\max }\right)^{1 / 2}
\end{gathered}
$$

of the absorption maximum. For a system with harmonic nuclear motion, the energy of activation $\Delta E^{*}$ is related to the energy of the IT band maximum $\left(\nu_{\max }\right)$ as shown by eq 3 for the weak-electron-exchange coupling case. The magnitude of the delocalization can be obtained by a calculation of the delocalization parameter $\alpha^{2}$ and electronic coupling $H_{\mathrm{ab}}$ from eqs 4 and 5 . In eqs 4 and $5 \epsilon_{\max }$ is the extinction coefficient and $d$ is the donor-acceptor distance. The average value $(5.1 \AA)$ of $\mathrm{Fe}-\mathrm{Fe}$ distances in a series of dialkyl mixed-valence biferrocenium cations is used as the donor-acceptor distance..$^{7,15}$ Finally, the rate constant $\left(K_{\mathrm{et}}\right)$ of intramolecular electron transfer can be estimated from eq 6, where $\nu_{\text {et }}$ is the hopping frequency.

In common with most mixed-valence complexes, the mixed-valence compounds $18 \mathrm{a}, \mathrm{b}$ in $\mathrm{CH}_{2} \mathrm{Cl}_{2}$ have an IT band at 5050 and $4808 \mathrm{~cm}^{-1}$, respectively, which is not present for the neutral compound or dioxidized ion. The

(34) Kaluski, Z. L.; Struchkov, Yu. T.; Avoyan, R. L. Zh. Strukt. Khim. $1964,5,743$.

(35) Hush, N. S. Prog. Inorg. Chem. 1967, 8, 391.
Table 9. Absorption Maxima of IT Bands and Activation Parameters

\begin{tabular}{cccccccc}
\hline compd & $\nu_{\max }{ }^{a}$ & $\epsilon_{\max }{ }^{b}$ & $\begin{array}{c}\Delta \nu_{1 / 2} \\
(\text { obsd) }\end{array}$ & $\begin{array}{c}\Delta \nu_{1 / 2} \\
(\text { calcd) }\end{array}$ & $\alpha^{2}$ & $H_{\mathrm{ab}}$ & $10^{-12} K_{\mathrm{et}}$ \\
\hline 1 & 4762 & 980 & 3670 & 3240 & 0.0123 & 528 & 1.96 \\
16 & 4762 & 2880 & 2898 & 3317 & 0.0286 & 805 & 4.68 \\
$18 \mathrm{a}$ & 5050 & 2690 & 2170 & 3415 & 0.0188 & 692 & 2.31 \\
$18 b$ & 4808 & 2930 & 2150 & 3333 & 0.0214 & 703 & 3.26
\end{tabular}

${ }^{a}$ In $\mathrm{cm}^{-1},{ }^{b}$ In $\mathrm{M}^{-1} \mathrm{~cm}^{-1}$.

absorption maximum and activation parameters calculated from eqs 2-6 were collected in Table 9, together with those for other relevant compounds. An interesting finding is that the IT bands of interannular trimethylene-bridged biferrocenium cations 16 and $18 \mathrm{a}, \mathrm{b}$ are all sharper than what is expected on the basis of eq 2 . An agreement, to about $10 \%$, between $\Delta \nu_{1 / 2}$ (calcd) and $\Delta \nu_{1 / 2}$ (obsd) is usually taken as an indication that the Hush model is a satisfactory description of a localized mixed-valence system. In the case of 1 , a good agreement is observed. A sharper band is usually observed for a delocalized mixed-valence compound. For example, the IT band in the Creutz-Taube ion is about 6 times sharper than that predicted by eq $2 .^{36}$ The Hush model is derived for the high-temperature limit, the criterion for that limit being $2 R T>h \nu$, where $h \nu$ is the energy associated with a metal-ligand vibrational transition. For either a Fe(II) - or a Fe(III)-Cp vibration in 16 and $18 a, b$, at room temperature we are not at the hightemperature limit. Owing to the interannular trimethylene bridge, the $\mathrm{Fe}-\mathrm{Cp}$ vibrational energy in 16 and $18 \mathrm{a}, \mathrm{b}$ is higher than that in 1 . Possibly as a consequence of this fact, Hush's correlation of band energy with band width (at room temperature; eq 2) fails.

The extent of delocalization of the exchanging electron in mixed-valence cations can be estimated from the properties of their IT bands. A measure of delocalization in both the ground and excited states is $\alpha^{2}$, which can be calculated from eq 4. The experimental $\alpha^{2}$ value is the average of $\alpha^{2}$ values for the ground and excited states. If delocalization is small, the electronic wave functions used for overlap are relatively unperturbed, and $\alpha^{2}$ is a direct measure of delocalization in the ground state. As shown in Table 9, in all cases there is good evidence for localized valences and delocalization from $\mathrm{Fe}$ (II) to $\mathrm{Fe}$ (III) is less than $3 \%$.

From the rate constants illustrated in Table 9, the electron-transfer rates in 16 and $18 a, b$ are faster than that in biferrocenium triiodide (1). This difference in rate clearly indicates that the interannular bridge also plays an important role in controlling the rate of electron transfer.

${ }^{57}$ Fe Mössba uer Characteristics. Variable-temperature Mössbauer spectra were run for compounds 18a,b. The various absorption peaks in each spectrum were fitted to Lorentzian lines, and the resulting fitting parameters are summarized in Table 10 . The variable-temperature Mössbauer spectra for 18a are shown in Figure 5. The features in these spectra include two doublets which have the same area, as deduced by a least-squares fitting. This pattern of two doublets is expected for a mixed-valence biferrocenium cation which is valence-trapped on the time scale of the Mössbauer technique (electron-transfer rate less than $\sim 10^{7} \mathrm{~s}^{-1}$ ). The valence-trapped electronic structure is also observed for compound 18b. As illustrated

(36) Creutz, C.; Taube, H. J. Am. Chem. Soc. 1973, 95, 1086. 
Table 10. ${ }^{57} \mathrm{Fe}$ Mössbauer Least-Squares-Fitting Parameters

\begin{tabular}{ccccc}
\hline compd & $T(\mathrm{~K})$ & $\Delta E_{\mathrm{Q}}(\mathrm{mm} / \mathrm{s})^{a}$ & $\delta(\mathrm{mm} / \mathrm{s})^{b}$ & $\Gamma(\mathrm{mm} / \mathrm{s})^{c}$ \\
\hline 1 & 300 & 1.908 & 0.486 & $0.260,0.276$ \\
& & 0.386 & 0.487 & $0.282,0.292$ \\
$18 \mathrm{a}$ & 300 & 1.864 & 0.356 & $0.259,0.284$ \\
& & 1.273 & 0.368 & $0.285,0.302$ \\
& 200 & 1.869 & 0.401 & $0.253,0.265$ \\
& & 1.334 & 0.411 & $0.274,0.295$ \\
& 150 & 1.874 & 0.416 & $0.246,0.255$ \\
& & 1.357 & 0.428 & $0.280,0.291$ \\
& 77 & 1.878 & 0.434 & $0.253,0.254$ \\
186 & 300 & 1.371 & 0.448 & $0.285,0.289$ \\
& & 1.766 & 0.370 & $0.357,0.379$ \\
& 77 & 1.079 & 0.382 & $0.438,0.438$ \\
& & 1.825 & 0.437 & $0.328,0.342$ \\
19 & 300 & 1.146 & 0.456 & $0.442,0.431$ \\
20 & 300 & 0.148 & 0.382 & $0.314,0.472$ \\
& 77 & 0.551 & 0.321 & $0.298,0.332$ \\
21 & 300 & 0.936 & 0.404 & $0.568,1.168$ \\
& 77 & 1.023 & 0.425 & $0.341,0.312$ \\
22 & 300 & 0.627 & 0.514 & $0.285,0.274$ \\
& 77 & 0.738 & 0.256 & $0.282,0.309$ \\
23 & 300 & 2.111 & 0.347 & $0.382,0.533$ \\
& & 0.771 & 0.434 & $0.225,0.229$ \\
& 77 & 2.137 & 0.418 & $0.265,0.295$ \\
& & 0.841 & 0.521 & $0.239,0.245$ \\
& & 0.514 & $0.284,0.291$
\end{tabular}

${ }^{a}$ Quadrupole splitting. ${ }^{b}$ Isomer shift. ${ }^{c}$ Full width at half-height taken from the least-squares fitting program. The width for the line at more positive velocity is listed first for the doublet.

(A)

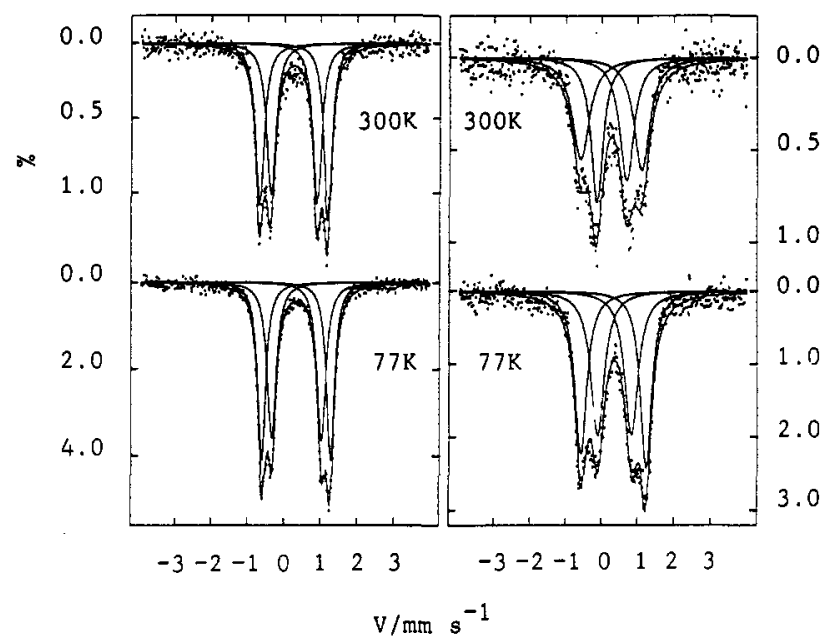

Figure 5. ${ }^{57} \mathrm{Fe}$ Mössbauer spectra for $18 \mathrm{a}(\mathrm{A})$ and $18 \mathrm{~b}$ (B).

in Figure 5 , the features in the $300 \mathrm{~K}$ spectrum of $18 \mathrm{~b}$ are two doublets, one with a quadrupole splitting $\left(\Delta E_{\mathrm{Q}}\right)$ of $1.177 \mathrm{~mm} \mathrm{~s}^{-1}\left(\mathrm{Fe}\right.$ (III) site) and the other with $\Delta E_{\mathrm{Q}}=1.826$ $\mathrm{mm} \mathrm{s}^{-1}$ (Fe(II) site). Furthermore, both doublets have the same area by a least-squares fitting to Lorentzian line shapes.

As mentioned in the Introduction, compound 16 has a delocalized electronic structure above $77 \mathrm{~K}$ (electrontransfer rate greater than $\sim 10^{7} \mathrm{~s}^{-1}$ ). ${ }^{21,24}$ Thus, there is a dramatic difference in electron-transfer rates between 16 and 18. We believe that this is mainly due to the differences in coplanarity of the two $\mathrm{Cp}$ rings in the fulvalenide ligand in 16 and 18. The single-crystal X-ray determinations of $18 \mathbf{a}, \mathbf{b}$ indicate that the $\pi$ interaction between the two ferrocenyl moieties is destroyed as the linking bond of the two ferrocenyl units is twisted.

The Mössbauer results also lead us to conclude that compound 16 has a localized electronic ground state at 4.2 $\mathrm{K}$ (electron-transfer rate $<10^{7} \mathrm{~s}^{-1}$ ). However, it is necessary
Chart 2
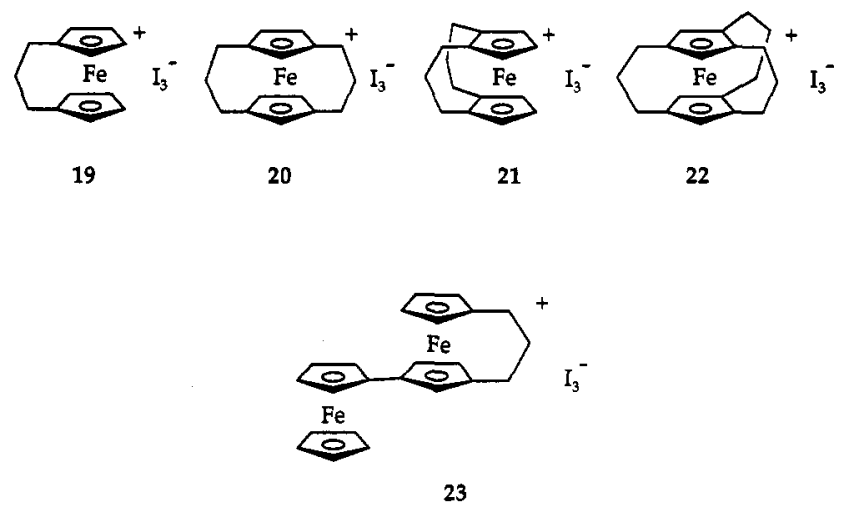

to discuss why the ferrocenium moiety in 16 and $18 \mathrm{a}, \mathrm{b}$ has an unusually large quadrupole splitting of $\sim 1.3 \mathrm{~mm} \mathrm{~s}^{-1}$. In general, ferrocenyl groups (electronic ground state ${ }^{1} \mathrm{~A}_{1 \mathrm{~g}}$ ) give spectra characterized by large quadrupole splitting in the range of $2.0-2.2 \mathrm{~mm} \mathrm{~s}^{-1}$, while the spectra of ferrocenium cations (electronic ground state ${ }^{2} \mathrm{E}_{2 g}$ ) are characterized by small or vanishing quadrupole splitting. ${ }^{37}$ In the case of a Mössbauer localized mixed-valence biferrocenium cation, the value of $\Delta E_{\mathrm{Q}}$ in the ferrocenyl moiety is slightly smaller than what is expected for a Fe(II) ferrocenyl unit and the Fe(III) ferrocenium moiety has a slightly larger $\Delta E_{Q}$ value. Of course, this is a reflection of the admixture of $\mathrm{Fe}$ (II) and $\mathrm{Fe}$ (III) moieties into the ground state. ${ }^{7,37}$ For instance, compound 1 gives a $300 \mathrm{~K}$ Mössbauer spectrum with two doublets $\left(\Delta E_{\mathrm{Q}}=\right.$ 1.908 and $\left.0.386 \mathrm{~mm} \mathrm{~s}^{-1}\right) .^{7}$ Temperature-dependent Mössbauer delocalized mixed-valence biferrocenium cations (for example, compound 2) at temperatures below transition temperature show two doublets, one for $\mathrm{Fe}$ (II) and the other for $\mathrm{Fe}$ (III) sites. ${ }^{7,8}$ Increasing the temperature causes the two doublets to move together and eventually to become a single average-valence $\operatorname{doublet}\left(\Delta E_{\mathrm{Q}} \approx 1.1 \mathrm{~mm}\right.$ $\mathbf{s}^{-1}$ ) at transition temperature. In our case, the unusually large $\Delta E_{Q}$ value cannot be explained in terms of an admixture of $\mathrm{Fe}(\mathrm{II})$ and $\mathrm{Fe}$ (III) electronic descriptions. For compounds $18 \mathbf{a}, \mathbf{b}$, as the temperature of the sample is increased from 77 to $300 \mathrm{~K}$, it is interesting to find that the two doublets do not move together. To explain this unusual observation, we have prepared the series of model compounds $19-23$ to understand the influence of an interannular trimethylene bridge on the electronic ground state of the ferrocenium cation (Chart 2). Surprisingly, a large quadrupole splitting is observed in the Mössbauer spectra of 20-22 at $300 \mathrm{~K}$. The $\Delta E_{\mathrm{Q}}$ values of 19-22 are $0.148,0.541,0.936$, and $0.627 \mathrm{~mm} \mathrm{~s}^{-1}$, respectively. For the asymmetric mixed-valence compound 23 , the features in the $300 \mathrm{~K}$ Mössbauer spectrum are two doublets, one for the $\mathrm{Fe}$ (II) metallocene $\left(\Delta E_{\mathrm{Q}}=2.111 \mathrm{~mm} \mathrm{~s}^{-1}\right)$ and the other for the Fe(III) metallocene $\left(\Delta E_{Q}=0.771 \mathrm{~mm} \mathrm{~s}^{-1}\right){ }^{38}$ The Mössbauer studies clearly indicate that the electronic ground state of the Fe(III) metallocene in 19-23 is not a pure ${ }^{2} \mathrm{E}_{2 \mathrm{~g}}$ state. In compounds $19-23$, the $\mathrm{Cp}$ rings are tilted from the parallel geometry for ferrocenium. Bending back the $\mathrm{Cp}$ rings leads to an increase of $\mathrm{d}_{x^{2}-y^{2}}, \mathrm{~d}_{x y}$-ring overlap. In other words, the metal nonbonding orbitals start to interact with the ligand $\pi$ orbitals. Under these circumstances the iron ions lose some degree of their Fe-

(37) Morrison, W. H., Jr.; Hendrickson, D. N. Inorg. Chem. 1975, 14, 2331.

(38) Dong, T.-Y.; Lin, H. M. J. Organomet. Chem. 1992, 426, 369. 
(III) character, and this results in an increase in $\Delta E_{Q}$ because each iron ion is closer to $\mathrm{Fe}$ (II) in its properties. This is what we observe for compounds $16,18 a, b$, and 19-23.

\section{Conclusions and Comments}

Mössbauer spectra indicating the presence of localized electronic structure have been observed for $18 \mathbf{a}, \mathbf{b}$. The energy and the line shape of the IT band clearly indicate that the intramolecular electron-transfer rates in $18 \mathrm{a}$ and $18 \mathrm{~b}$ in solution are greater than in the solid state. In the solid state, the rate of electron transfer for a given mixedvalence cation is influenced by various structural factors and lattice dynamics, 6,7 including the electronic and vibronic couplings between two metal ions, the nature of the counterion, and cation-anion interactions. ${ }^{11,12}$ In general, the electron-transfer rate for a given mixed-valence biferrocenium cation in the solution state is greater than that in the solid state. In the solid state, we have demonstrated that relatively minor perturbations caused by interactions between neighboring cations and anions have pronounced effects on the electron transfer. ${ }^{18}$ In solution, the manner in which the mixed-valence cation is solvated also influences the rate of intramolecular electron transfer. If ion pairing is present, the anion must move rapidly so as not to limit the rate of intramolecular electron transfer. However, in the case of $18 \mathbf{a}, \mathbf{b}$, we believe that the most important factor determining the difference of intramolecular electron-transfer rate between the solution and the solid state is the degree of coplanarity between two $\mathrm{Cp}$ rings in the fulvalenide bridge. As is evident in the section Electrochemical Results, in the solution state the two $\mathrm{Cp}$ rings of the fulvalenide bridge in the cations of $17 a, b$ are coplanar. However, the two Cp rings of the fulvalenide bridge in $18 \mathbf{a}, \mathbf{b}$ are not crystallographically coplanar.

We have also demonstrated that the perturbations caused by interannular trimethylene bridges have dramatic influences on the electronic structure in a series of model compounds. The cation in each of compounds 18-23 serves as a very sensitive probe of the microscopic structure of the electronic state. Characterization of the electronic ground state in these cations by magnetic susceptibility and EPR and an investigation of the counterion effects on the rate of electron transfer in the solid state are underway. Calculations of the theoretical values of $\Delta E_{\mathrm{Q}}$ in the cations of 18-23 are also in progress.

Acknowledgment. Our work was generously supported by the National Science Council, National Sun Yatsen University, and Academia Sinica. We gratefully acknowledge this support.

Supplementary Material Available: Complete tables of positional parameters, bond lengths and angles, and thermal parameters for $17 \mathrm{a}, \mathrm{b}$ and $18 \mathrm{a}, \mathrm{b}$ ( $48 \mathrm{pages}$ ). Ordering information is given on any current masthead page.

OM940127V 